\title{
Dynamics of heteropolymers in dilute solution: effective equation of motion and relaxation spectrum
}

\author{
Jiunn-Ren Roan and E. I. Shakhnovich \\ Chemistry Department, 12 Oxford Street, Cambridge, MA 02138, USA
}

(March 27, 2018)

\begin{abstract}
The dynamics of a heteropolymer chain in solution is studied in the limit of long chain length. Using functional integral representation we derive an effective equation of motion, in which the heterogeneity of the chain manifests itself as a time-dependent excluded volume effect. At the mean field level, the heteropolymer chain is therefore dynamically equivalent to a homopolymer chain with both time-independent and time-dependent excluded volume effects. The perturbed relaxation spectrum is also calculated. We find that heterogeneity also renormalizes the relaxation spectrum. However, we find, to the lowest order in heterogeneity, that the relaxation spectrum does not exhibit any dynamic freezing, at the point when static (equilibrium) "freezing" transition occurs in heteropolymer. Namely, the breaking of fluctuation-dissipation theorem (FDT) proposed for spin glass dynamics does not have dynamic effect in heteropolymer, as far as relaxation spectrum is concerned. The implication of this result is discussed.
\end{abstract}




\section{INTRODUCTION}

Although systems without quenched randomness usually provide useful information and serve as the first step of our understanding of condensed phase, there are situations in which quenched randomness brings entirely new phenomena. Flux line pinning in type II superconductors, critical to the practical application of superconductors in high field magnets, is a manifestation of the embedded quenched impurities [1]. In the mixture of ${ }^{3} \mathrm{He}$ and ${ }^{4} \mathrm{He}$, quenched randomness introduced by porous media (e.g. aerogels) shifts the tricritical point and extends the range of superfluid phase [2]. The interesting properties (e.g. the onset of remanence effects below a freezing temperature) of dilute magnets, modeled by "spin glass" [3], are consequences of the magnetic impurities. These are just a few examples of the new phenomena brought by quenched randomness. Study of these phenomena constitutes an important part of modern condensed phase physics.

Theoretical progress in the study of these systems, especially spin glass systems, also has brought impetus to other, somewhat distant, fields. For example, it is shown that there is an interesting analogy between some models developed for spin glasses and the statistical properties of polymers with quenched randomness (realized as heterogeneity of the polymer) [4]. This analogy has attracted much attention in recent years due to its possible biophysical applications. As much work has been focused on the static (thermodynamic) consequences of this analogy, we shall discuss in this paper its dynamic consequence.

One of the general methods used to study systems with quenched randomness is the so-called replica method [3, 5]. Because the randomness is quenched, thermal equilibrium is established without thermally equilibrating with the randomness. Therefore, to calculate the thermodynamic quantities for a system with quenched randomness one has to calculate the average of the free energy over the distribution of the randomness,

$$
\left\langle-k_{B} T \ln Z\right\rangle
$$

The mathematical obstacle lies in the difficulty in calculating the average of a logarithmic 
function depending on the randomness. Replica method circumvents this obstacle by using the identity

$$
\ln Z=\lim _{n \rightarrow 0} \frac{Z^{n}-1}{n}
$$

Replica method has been very useful and successful, though not free from controversy. With its success and popularity in spin glasses, it is not surprising that the study of heteropolymers along the line of the above-mentioned analogy often, if not exclusively, uses this method to draw thermodynamic conclusions.

Besides the replica method, there exist other useful theoretical methods to study systems with quenched disorder [3]. Since we are interested in the dynamics of heteropolymers, among these methods the Langevin dynamic method [3:6] is the most relevant one. This method does not use the unphysical replica limit $n \rightarrow 0$ to calculate thermodynamic quantities. In Ref. [6] it is shown that the functional integral formalism developed by Martin, Siggia, and Rose for classical statistical dynamics [7] allows average over the quenched randomness without using the replica method. It is also shown that this formalism is capable of giving dynamic information as well as statics. Therefore, although it generally involves more complicated techniques, it is worthwhile to study systems with quenched randomness using this method. In spin glasses there have been some studies using this approach [3, 8, 9]. On the side of heteropolymers, however, to the best of our knowledge, a dynamic study similar to these studies is still lacking. While the static analogy between spin glasses and heteropolymers has been very useful, a similar dynamic study is necessary if one wants to use this analogy to address dynamic issues.

The purpose of this paper is to study the dynamics of single heteropolymer chain, i.e. dilute heteropolymer solution. It is well known that the dynamic study of polymer solutions, especially dilute solutions, must take into account the hydrodynamic effect in order to make the prediction experimentally relevant [10 [12]. Besides hydrodynamic effect, the heterogeneity of the polymer chain presumably introduces further effect. One expects that the dynamics of the heteropolymer chain will depend on these two effects as well as the 
usual excluded volume effect. Here we investigate the importance of the heterogeneity to the chain dynamics.

The dynamics of dilute polymer solution has been extensively studied [10,12]. Dynamic quantities such as diffusion constant [13], time-correlation functions [14], intrinsic viscosity [15], initial decay rate of the dynamical scattering [16], and relaxation spectrum [17] have been calculated. Among these dynamic quantities we are most interested in the relaxation spectrum because, in the context of Ref. [4], i.e. protein dynamics, the relaxation spectrum is the most relevant quantity with potential application to the protein folding problem. In spite of the close analogy between models of spin glasses and heteropolymers at static level [4], we find that this analogy is not as complete at dynamic level. While there have been attempts to study heteropolymer dynamics at the phenomenological level of the hypothetical dynamics of the Random Energy Model (REM) [18], the present microscopic analysis suggests that such analogy may not be justified when dynamic issues are tackled.

The structure of this paper is as follows. In Sec. II we define the model Hamiltonian and the dynamic equation of the system. We discuss the physics of the model Hamiltonian and the dynamic equation. Assumptions made are also addressed. In Sec. III we derive the mean field equation of motion for the chain in the limit of long chain length. This effective equation of motion can be further simplified when the characteristic time scale of the solvent is much shorter than the characteristic time scale of the chain. An effective Hamiltonian results from this effective equation. It is shown that the effective Hamiltonian contains a memory term, and, hence, a time-dependent excluded volume interaction. In Sec. IVA we calculate the perturbed relaxation spectrum for the chain, to the lowest order in excluded volume interaction, hydrodynamic interaction, and heterogeneity. We find that, as long as the model Hamiltonian and dynamic equation are valid, the relaxation spectrum does not depend on the final equilibrium state of the chain. In other words, in spite of the static analogy between spin glass and heteropolymer mentioned above, the possible breaking of FDT, due to the degeneracy of the ground states, found in the mean field dynamics of spin glass [3,8] does not have corresponding dynamical effect, at least to order of our calculation, 
in the mean field dynamics of heteropolymer. In Sec. $\mathrm{V}$ we discuss the analogy of spin glass and heteropolymer, using the well-known correspondence between magnetic system and self-avoiding random walk. We will see that, when the kinetic equations are compared, it is clear that the analogy is not very helpful, because the dynamic variables used are completely

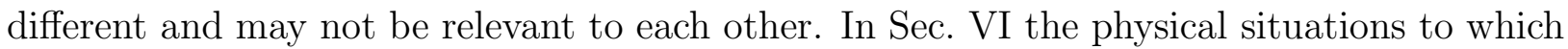
our model may be applicable are discussed. We also give a brief discussion of approximations involved and the limitation of the results. In App. A we provide an alternative derivation of the conventional (Kirkwood) approach used in the dynamic study of dilute polymer solution. App. B gives the detailed derivation of the mean field approximation sketched in Sec. III. In App. Q we detail the calculation leading to the result in Sec. IVA.

\section{THE MODEL}

\section{A. Hamiltonian}

Consider a heteropolymer chain with conformation specified by the coordinates of $N$ monomers, $\mathbf{r}_{0}, \cdots, \mathbf{r}_{N-1}$. The chain can be described by the following Hamiltonian,

$$
\begin{gathered}
H=H_{0}+H_{R} \\
H_{0} \equiv \sum_{i=0}^{N-1} \frac{1}{2}\left(\mathbf{r}_{i+1}-\mathbf{r}_{i}\right)^{2} \\
H_{R} \equiv \frac{1}{2} \sum_{i \neq j} B_{i j} U\left(\mathbf{r}_{i}-\mathbf{r}_{j}\right),
\end{gathered}
$$

where $B_{i j}$ is a Gaussian random number centered at $B_{0}$ with variance $B^{2}(B>0)$,

$$
P\left(B_{i j}\right)=\frac{1}{\sqrt{2 \pi B^{2}}} \exp \left[-\frac{\left(B_{i j}-B_{0}\right)^{2}}{2 B^{2}}\right],
$$

In Eqs. (2.1) $H_{0}$ is the Hamiltonian used in the bead-spring chain model (Rouse model) [11], where, for convenience, all the spring constants have been taken to be unity (see below). The chain connectivity of the polymer is described by this term. $H_{R}$ simulates the excluded 
volume effect. The excluded volume interaction $U\left(\mathbf{r}_{i}-\mathbf{r}_{j}\right)$ between monomer $i$ and monomer $j$ is Gaussian-modulated by $B_{i j}$. This modulation confers the chain heterogeneity. When $B \rightarrow 0$ the excluded volume interactions are "monochromatically" modulated by $B_{0}$ and the chain becomes homogeneous. At this extreme, the Hamiltonian, Eq. (2.1), reduces to the usual Edwards Hamiltonian [12],

$$
H_{E} \equiv \sum_{i=0}^{N-1} \frac{1}{2}\left(\mathbf{r}_{i+1}-\mathbf{r}_{i}\right)^{2}+\frac{B_{0}}{2} \sum_{i \neq j} U\left(\mathbf{r}_{i}-\mathbf{r}_{j}\right)
$$

On the other hand, a nonvanishing $B$ means that there exist various monomer-monomer interactions, and the chain is essentially heterogeneous. Thus $B$ is a measure of chain heterogeneity and will be called heterogeneity parameter. $B_{0}$ is the usual excluded volume parameter measuring the strength of excluded volume effect. Presumably, $B$ depends only on the heterogeneity (dispersity) of polymer, while $B_{0}$ is generally a function of temperature and solvent quality.

The excluded volume parameter $B_{0}$ will be taken to be positive throughout this paper. A negative $B_{0}$ gives attractive monomer-monomer interactions, which requires inclusion of repulsive three-body interactions in order to render the theory stable [19,20]. To simplify the matter, we will confine ourselves to positive $B_{0}$, i.e. a heteropolymer chain in good solvent.

The conventional use of the excluded volume interaction $U(\mathbf{r})$ is the hard-core repulsion $\delta(\mathbf{r})[12,19,20$. To derive the effective equation of motion at the mean field level in a more general form, we will not adopt this convention for $U(\mathbf{r})$ until Sec. IV A, where the perturbed relaxation spectrum is calculated. Before Sec. IVA the only assumption on $U(\mathbf{r})$ will be translational invariance: $U\left(\mathbf{r}, \mathbf{r}^{\prime}\right)=U\left(\mathbf{r}-\mathbf{r}^{\prime}\right)=U\left(\mathbf{r}^{\prime}-\mathbf{r}\right)$.

Obviously, the Hamiltonian $H_{0}$ ignores the possible variation of spring constants. Presumably, this variation exists when the chain is heterogeneous. Since our concern here is the effect of random excluded volume interactions, we do not consider this variation in spring constants. With this simplification, by a properly chosen length scale, we can always set all spring constants to be unity, as is done in (2.1b). 
The statics of Hamiltonian (2.1) has been studied in Refs. [4], within the context of biopolymer and protein folding. These works share a number of techniques with spin-glass models. In the past two decades there has been much progress in the theories of spin-glass, in both statics and dynamics [3]. However, there has never been any systematic microscopic study on the dynamics of Hamiltonian (2.1).

\section{B. Dynamics}

Starting with the Hamiltonian (2.1), for dynamic study, we consider the coupled Langevin equations proposed by Oono and Freed [21]:

$$
\begin{gathered}
\frac{\partial}{\partial t} \mathbf{r}_{i}(t)=-\frac{1}{\zeta_{0}} \frac{\partial H}{\partial \mathbf{r}_{i}(t)}+g_{0} \mathbf{u}\left(\mathbf{r}_{i}(t), t\right)+\boldsymbol{\theta}_{0 i}(t) \\
\left\langle\boldsymbol{\theta}_{0 i}(t)\right\rangle=0, \quad\left\langle\boldsymbol{\theta}_{0 i}(t) \boldsymbol{\theta}_{0 j}\left(t^{\prime}\right)\right\rangle=\frac{2}{\zeta_{0}} \delta\left(t-t^{\prime}\right) \delta_{i j} \mathbf{1} \\
\frac{\partial}{\partial t} \mathbf{u}(\mathbf{r}, t)=\nu_{0} \Delta \mathbf{u}(\mathbf{r}, t)-\frac{g_{0}}{\rho_{0}} \sum_{i=0}^{N-1} \frac{\partial H}{\partial \mathbf{r}_{i}(t)} \delta\left(\mathbf{r}-\mathbf{r}_{i}(t)\right)-\frac{1}{\rho_{0}} \boldsymbol{\nabla} p+\boldsymbol{\xi}_{0}(\mathbf{r}, t), \\
\left\langle\boldsymbol{\xi}_{0}(\mathbf{r}, t)\right\rangle=0, \quad\left\langle\boldsymbol{\xi}_{0}(\mathbf{r}, t) \boldsymbol{\xi}_{0}\left(\mathbf{r}^{\prime}, t^{\prime}\right)\right\rangle=-\frac{2 \eta_{0}}{\rho_{0}^{2}} \Delta \delta\left(\mathbf{r}-\mathbf{r}^{\prime}\right) \delta\left(t-t^{\prime}\right) \mathbf{1}
\end{gathered}
$$

where 1 is a unit tensor. In these equations the kinetic coefficient $\zeta_{0}^{-1}$ and kinematic viscosity $\nu_{0}$ set the time scales for polymer and solvent molecules, respectively. $g_{0}$ is a measure of the coupling strength between monomers and solvent molecules. (We also ignore the possible variation of these couplings due to the chain heterogeneity.) Langevin noise $\boldsymbol{\theta}_{0 i}(t)$ gives monomer $i$ a random velocity at time $t$. Noise $\boldsymbol{\xi}_{0}(\mathbf{r}, t)$ is the random acceleration of the solvent velocity field at position $\mathbf{r}$ and time $t$. Dynamic viscosity $\eta_{0}$ is, as usual, related to the solvent density $\rho_{0}$ by $\eta_{0}=\rho_{0} \nu_{0}$. The hydrostatic pressure $p$ ensures the incompressibility of the solvent [22]. This condition enables us to consider only the transverse component of solvent velocity field. Since no confusion will be arisen, hereafter, we will denote the transverse component of solvent velocity by the same symbol $\mathbf{u}$. 
Eq. (2.4a) is a relaxation dynamic equation, with mode-coupling term $g_{0} \mathbf{u}$, for the chain. Eq. (2.4d) is the Stokes approximation of the Navier-Stokes equation for solvent velocity field $\mathbf{u}$, augmented by the forces exerted by monomers [22]. We will assume that the spatial extension of the system is infinite so that we can ignore the boundary condition. The problem related to the non-applicability of the Navier-Stokes equation in an unbounded space will be also ignored [23,24. Eqs. (2.4) are valid as long as the Reynolds number is small.

In the conventional theory of Brownian motion the effect of surrounding solvent molecules is taken into account by the Langevin noises. In polymer dynamics the commonly used approach (Kirkwood's approach) is essentially based on this viewpoint [10 12]. This approach treats each monomer as a point source of frictional force and lumps up the effect of solvent molecules in the Langevin noises. However, as argued by Oono [12,25], this picture is not self-consistent because the monomers are not legitimate Brownian particles, even if the polymer as a whole can be treated like a Brownian object. In contrast to Kirkwood's approach, the coupled Langevin equations Eqs. (2.4) proposed by Oono and Freed explicitly introduces solvent velocity field. In this approach the Langevin noises $\boldsymbol{\theta}_{0}$ and $\boldsymbol{\xi}_{0}$ have to be re-interpreted as coming from the coarse-graining procedures used to derive these equations, separately, from the more fundamental, microscopic, equations, and the coupling between polymer and solvent is introduced only after the respective coarse-graining procedures have been performed. A proper term for these equations is "kinetic equation" 26]. Therefore, we will simply call Eqs. (2.4) Oono-Freed kinetic equation.

In the Oono-Freed kinetic equation the energy scale has been chosen as $k_{B} T=1$. The correlation of Langevin noises chosen here, Eqs. (2.4b) and (2.4d) guarantees that the system will eventually approach to the equilibrium state determined by the statics of Hamiltonian (2.1).

Note that the second term on the right side of Eq. $(2.4 \mathrm{c})$ is the frictional forces exerted by the monomers, which are point sources of frictional force, same as in Kirkwood's approach. In fact, there is a close relationship between these two approaches. It has been shown that, to the lowest nontrivial order (i.e. to the lowest order in the excluded-volume parameter $B_{0}$ 
and hydrodynamic coupling parameter $g_{0}$ ) and within Markovian approximation for solvent velocity field, when the solvent velocity field is projected out, the Fokker-Planck equation for the kinetic equation Eqs. (2.4) reduces to the conventional Kirkwood diffusion equation [12,16]. A simplified proof of this reduction is given in App. A. These equations are, in this sense, more fundamental than the commonly used Kirkwood diffusion equation, upon which most dynamic study of dilute polymer solution are built. Therefore, a full dynamic study should start from Eqs. (2.4).

Using the same spirit of the proof in App. A, we first project out the velocity field from the kinetic equation. This is done by formally solving Eq. (2.4c),

$$
\begin{aligned}
\mathbf{u}(\mathbf{r}, t) & =\mathbf{u}_{0}(\mathbf{r}, t)+\mathbf{u}_{R}(\mathbf{r}, t) \\
\mathbf{u}_{0}(\mathbf{r}, t) & \equiv \int_{\mathbf{k}} e^{i \mathbf{k} \cdot \mathbf{r}} \mathbf{u}_{0}(\mathbf{k}, t) \\
& =\int_{\mathbf{k}} e^{i \mathbf{k} \cdot \mathbf{r}} \int_{-\infty}^{t} d t^{\prime} e^{-\nu_{0} k^{2}\left(t-t^{\prime}\right)} \mathbf{P}^{T}(\hat{\mathbf{k}}) \cdot\left[\boldsymbol{\xi}_{0}\left(\mathbf{k}, t^{\prime}\right)-\frac{g_{0}}{\rho_{0}} \sum_{i=0}^{N-1} \frac{\partial H_{0}}{\partial \mathbf{r}_{i}\left(t^{\prime}\right)} e^{-i \mathbf{k} \cdot \mathbf{r}_{i}\left(t^{\prime}\right)}\right] \\
\mathbf{u}_{R}(\mathbf{r}, t) & \equiv \int_{\mathbf{k}} e^{i \mathbf{k} \cdot \mathbf{r}} \mathbf{u}_{0}(\mathbf{k}, t) \\
& =\int_{\mathbf{k}} e^{i \mathbf{k} \cdot \mathbf{r}} \int_{-\infty}^{t} d t^{\prime} e^{-\nu_{0} k^{2}\left(t-t^{\prime}\right)} \mathbf{P}^{T}(\hat{\mathbf{k}}) \cdot\left[-\frac{g_{0}}{\rho_{0}} \sum_{i=0}^{N-1} \frac{\partial H_{R}}{\partial \mathbf{r}_{i}\left(t^{\prime}\right)} e^{-i \mathbf{k} \cdot \mathbf{r}_{i}\left(t^{\prime}\right)}\right]
\end{aligned}
$$

In Eqs. 2.5b) and (2.5q) $\mathbf{P}^{T}(\hat{\mathbf{k}})$ is the transverse (along $\hat{\mathbf{k}} \equiv \mathbf{k} / k$ direction) projection operator,

$$
\mathbf{P}^{T}(\hat{\mathbf{k}}) \equiv \mathbf{1}-\hat{\mathbf{k}} \hat{\mathbf{k}}
$$

and

$$
\int_{\mathbf{k}} \equiv \int \frac{d^{d} \mathbf{k}}{(2 \pi)^{d}}
$$

( $d$ is the spatial dimensionality). The Fourier-transformed random acceleration,

$$
\boldsymbol{\xi}(\mathbf{r}, t) \equiv \int_{\mathbf{k}} e^{i \mathbf{k} \cdot \mathbf{r}} \boldsymbol{\xi}(\mathbf{k}, t)
$$

is Gaussian and is correlated according to

$$
\left\langle\boldsymbol{\xi}^{\perp}(\mathbf{k}, t) \boldsymbol{\xi}^{\perp}\left(\mathbf{k}^{\prime}, t^{\prime}\right)\right\rangle=\mathbf{P}^{T}(\hat{\mathbf{k}}) \frac{2 \eta_{0}}{\rho_{0}^{2}} k^{2} \delta\left(\mathbf{k}+\mathbf{k}^{\prime}\right) \delta\left(t-t^{\prime}\right)
$$


where $\perp$ denotes the transverse component, $\boldsymbol{\xi}^{\perp}(\mathbf{k}, t) \equiv \mathbf{P}^{T}(\hat{\mathbf{k}}) \cdot \boldsymbol{\xi}(\mathbf{k}, t)$.

Substituting solution Eq. 2.5) into Eq. (2.4a), the equation of motion for the chain becomes

$$
\frac{\partial}{\partial t} \mathbf{r}_{i}(t)=-\frac{1}{\zeta_{0}} \frac{\partial H_{0}}{\partial \mathbf{r}_{i}(t)}+g_{0} \mathbf{u}_{0}\left(\mathbf{r}_{i}(t), t\right)+\boldsymbol{\theta}_{0 i}(t)-\frac{1}{\zeta_{0}} \frac{\partial H_{R}}{\partial \mathbf{r}_{i}(t)}+g_{0} \mathbf{u}_{R}\left(\mathbf{r}_{i}(t), t\right),
$$

Eq. (2.10) is the starting equation of our dynamic study. Note that this equation has two stochatic terms: $g_{0} \mathbf{u}_{0}\left(\mathbf{r}_{i}(t), t\right)$ which contains $\boldsymbol{\xi}_{0}\left(\mathbf{k}, t^{\prime}\right)$, and $\boldsymbol{\theta}_{0 i}(t)$. In other words, the new Langevin noise is

$$
\boldsymbol{\theta}_{0 i}(t)+g_{0} \int_{\mathbf{k}} e^{i \mathbf{k} \cdot \mathbf{r}_{i}(t)} \int_{-\infty}^{t} d t^{\prime} e^{-\nu_{0} k^{2}\left(t-t^{\prime}\right)} \mathbf{P}^{T}(\hat{\mathbf{k}}) \cdot \boldsymbol{\xi}_{0}\left(\mathbf{k}, t^{\prime}\right)
$$

Obviously, this new Langevin noise is no longer $\delta$-correlated in time.

Eq. (2.10) is much more complicated than its spin-glass counterpart [3,8, ,9], because of the presence of the excluded volume interactions and the hydrodynamic coupling. In polymer dynamics, quenched randomness has been considered (for example, the mean field dynamics of directed polymer in random meida studied by Vilgis [27]), but the randomness is from external random potential rather than chemical dispersity. Also, equations similar to Eq. (2.10) in which hydrodynamic effect is included have been studied in the context

of homopolymers [12 14, 21, 25, 28, 29] and two dimensional membranes [30]. These studies, however, consider simpler situations in which either quenched randomness or excluded volume interaction is absent. Eq. (2.10) considers all three effects: quenched randomness, hydordynamic coupling, and excluded volume effect.

\section{MEAN FIELD APPROXIMATION AND THE EFFECTIVE EQUATION OF MOTION}

\section{A. Functional-integral representation}

The equation of motion, Eq. (2.10), is essentially a (nonlinear) stochastic differential equation with colored noise (Eq. (2.11) ] 31]. A standard and convenient method to study 
stochastic differential equations is to use functional integral formalism (Martin-Siggia-Rose (MSR) formalism) [7] 31]. The main idea is to write down the probability functional for the Langevin noises and make change of variables from these noises to dynamical variables. The stochastic differential equation itself is treated as a constraint, limiting the evolution of probability path. By introducing auxiliary fields conjugated to the dynamical variables, this constraint, in the form of a $\delta$ functional, can be written as a functional integral of these auxiliary fields. This leads to a probability functional in terms of the dynamical variables and their conjugated auxiliary fields. The auxiliary-field technique used in this formalism is similar to the technique used in the supersymmetry formalism of stochastic differential equations, in which the auxiliary fields are replaced by fermion fields [32,34].

Since the functional integral formalism for stochastic processes is already well documented, we would not go into any detail of it. Useful references are Refs. [7, 32, 33]. (The concise review in Ref. 35 is also helpful.) The MSR formalism has been widely used for dynamic study of condensed phase systems, for example, liquid-glass transition [35], turbulence [36], spin glass dynamics [8] and so on. Nevertheless, in polymer physics it is not often used. This is probably because that, in spite of its elegance, no new results have been obtained through using this formalism [37,38]. The study in Refs. [13,14, 28, 229] makes it clear that, for practical calculation, the MSR formalism does not achieve, at this point, better (higher order) results than the conventional approach [12,25]. The mathematical involvement makes higher order calculations insurmountable. However, for our purposes the MSR method is very useful as it allows to carry out quenched averaging in the most natural form (see below).

Using the MSR formalism, the generating functional for Eq. (2.10) is presented as:

$$
\begin{gathered}
Z_{R \theta_{0} \xi_{0}}=\int\left\{\mathcal{D} \mathbf{r}_{i}(t)\right\}\left\{\mathcal{D} \hat{\mathbf{r}}_{i}(t)\right\} J\left(\left\{\mathbf{r}_{i}\right\}\right) e^{L_{0}+L_{R}} \\
L_{0}=\int_{-\infty}^{\infty} d t \sum_{i=0}^{N-1} i \hat{\mathbf{r}}_{i}(t) \cdot\left[\frac{\partial}{\partial t} \mathbf{r}_{i}(t)+\frac{1}{\zeta_{0}} \frac{\partial H_{0}}{\partial \mathbf{r}_{i}(t)}-g_{0} \mathbf{u}_{0}\left(\mathbf{r}_{i}(t), t\right)-\boldsymbol{\theta}_{0 i}(t)\right] \\
L_{R}=\int_{-\infty}^{\infty} d t \sum_{i=0}^{N-1} i \hat{\mathbf{r}}_{i}(t) \cdot\left[\frac{1}{\zeta_{0}} \frac{\partial H_{R}}{\partial \mathbf{r}_{i}(t)}-g_{0} \mathbf{u}_{R}\left(\mathbf{r}_{i}(t), t\right)\right]
\end{gathered}
$$


Note that the Langevin noises $\boldsymbol{\theta}_{0}$ and $\boldsymbol{\xi}_{0}$ are now in $L_{0}$, while the quenched heterogeneity, represented by $B_{i j}$, is in $L_{R}$. Since $\boldsymbol{\theta}_{0}, \boldsymbol{\xi}_{0}$ and $B_{i j}$ are not coupled, we can take average of $Z_{R \theta_{0} \xi_{0}}$ over these random quantities separately.

The Jacobian $J\left(\left\{\mathbf{r}_{i}\right\}\right)$ in Eq. (3.1) is associated with the change of variables from Langevin noises to dynamical variables (mentioned above). Because of the following two reasons, we will drop the Jacobian from our calculation from now on. Firstly, the specific form of this Jacobian depends on the time-discretization scheme used when the functional integral representation is written down [39]. (For Stratonovich scheme Refs. [40,41] work out the detailed form.) Although it is not uniquely defined, one can get correct result provided one consistently uses the same discretization scheme 42]. Secondly, and more importantly, the Jacobian depends on the dynamical variables $\left\{\mathbf{r}_{i}\right\}$, not on the auxiliary variables $\left\{\hat{\mathbf{r}}_{i}\right\}$. As we can see from Eqs. (3.2) and (3.3) the equation of motion, Eq. (2.10), is always coupled to the auxiliary fields $\left\{\hat{\mathbf{r}}_{i}\right\}$. This will still be true for the effective equation of motion that we seek for. Therefore, whatever form the Jacobian has, or, whether it also gets averaged when we perform averages over $B_{i j}, \boldsymbol{\theta}_{0}$ and $\boldsymbol{\xi}_{0}$ is not important. We do not need it anyway.

In (3.1) only the auxiliary fields $\left\{\hat{\mathbf{r}}_{i}\right\}$ are introduced. In principle, for the Oono-Freed kinetic equation (2.4), there should also be auxiliary fields $\{\hat{\mathbf{u}}\}$, as in Ref. [13, [4], 28, 229]. Since we are not interested in the solvent velocity fields, $\mathbf{u}$, and have eliminated it from the kinetic equation, there is no need to introduce an auxiliary variable $\hat{\mathbf{u}}$ for $\mathbf{u}$.

For computational convenience we define three operators,

$$
\boldsymbol{\Gamma}_{i j}\left(t, t^{\prime}\right) \equiv \frac{g_{0}^{2}}{\rho_{0}} \zeta_{0} \int_{\mathbf{k}} e^{-\nu_{0} k^{2}\left(t-t^{\prime}\right)} e^{i \mathbf{k} \cdot\left[\mathbf{r}_{i}(t)-\mathbf{r}_{j}\left(t^{\prime}\right)\right]} \mathbf{P}^{T}(\hat{\mathbf{k}})
$$

(a symmetric tensor),

$$
a_{i}(t) \equiv \frac{1}{2} \int d t^{\prime} \sum_{j=0}^{N-1} i \hat{\mathbf{r}}_{j}\left(t^{\prime}\right) \cdot\left[\mathbf{1} \delta_{i j} \delta\left(t-t^{\prime}-\epsilon\right) \theta\left(t-t^{\prime}\right)+\boldsymbol{\Gamma}_{j i}\left(t^{\prime}, t\right) \theta\left(t^{\prime}-t\right)\right] \cdot \nabla_{i}(t)
$$

(a scalar differential operator; $\epsilon$ being an infinitesimal positive number), and

$$
\mathcal{O}_{i j} \equiv \int d t\left[a_{i}(t) U\left(\mathbf{r}_{i}(t)-\mathbf{r}_{j}(t)\right)+a_{j}(t) U\left(\mathbf{r}_{j}(t)-\mathbf{r}_{i}(t)\right)\right]
$$


(symmetric in $i$ and $j$ ). Now we can rewrite $L_{R}$ in a more compact form

$$
L_{R}=\sum_{i \neq j} \frac{1}{\zeta_{0}} B_{i j} \mathcal{O}_{i j},
$$

The average over $B_{i j}$ can be easily done and gives

$$
\begin{aligned}
Z_{\theta_{0} \xi_{0}} & =\int\left\{\mathcal{D} \mathbf{r}_{i}(t)\right\}\left\{\mathcal{D} \hat{\mathbf{r}}_{i}(t)\right\} e^{L_{0}}\left\langle e^{L_{R}}\right\rangle_{B} \\
& \left.=\int\left\{\mathcal{D} \mathbf{r}_{i}(t)\right\}\right\}\left\{\mathcal{D} \hat{\mathbf{r}}_{i}(t)\right\} e^{L_{0}} \exp \left[\frac{B^{2}}{2 \zeta_{0}^{2}} \sum_{i \neq j} \mathcal{O}_{i j}^{2}+\frac{B_{0}}{\zeta_{0}} \sum_{i \neq j} \mathcal{O}_{i j}\right]
\end{aligned}
$$

\section{B. Mean field approximation}

The generating functional obtained in Sec. III A can be used to derive an effective equation of motion in the limit of long chain length $N \rightarrow \infty$. The same method is used to derive

an effective equation of motion for spin glass [8,9] and for directed polymers [27]. Although the algebra involeve here is more complicated, the resulting effective equation of motion share the same characteristics as the effective equation of motion obtained in Ref. [8,9].

The detailed calculation in the long chain limit can be found in App. B. The result is an effective Lagrangian:

$$
\begin{array}{rl}
L_{e}=L_{0}+\frac{B^{2}}{\zeta_{0}^{2}} \int d t d t^{\prime} d & 1 d 2 d 3 d 4\left[\left\langle\bar{A}\left(t, t^{\prime}, 1,3\right)\right\rangle \bar{B}\left(t, t^{\prime}, 2,4\right)+\left\langle\bar{B}\left(t, t^{\prime}, 2,4\right)\right\rangle \bar{A}\left(t, t^{\prime}, 1,3\right)\right. \\
+ & \left.2\left\langle\bar{C}\left(t, t^{\prime}, 1,3\right)\right\rangle \bar{D}\left(t, t^{\prime}, 2,4\right)\right] U(1-2) U(3-4) \\
+ & \frac{2 B_{0}}{\zeta_{0}} \int d t d t^{\prime} d 1 d 2 d 3 d 4\left[\left\langle\bar{C}\left(t, t^{\prime}, 1,3\right)\right\rangle \bar{B}\left(t, t^{\prime}, 2,4\right)\right. \\
+ & \left.\left\langle\bar{B}\left(t, t^{\prime}, 2,4\right)\right\rangle \bar{C}\left(t, t^{\prime}, 1,3\right)\right] \delta\left(t-t^{\prime}\right) U(1-2),
\end{array}
$$

where, for convenience, $\mathbf{R}_{1}, \mathbf{R}_{2}, \mathbf{R}_{3}, \mathbf{R}_{4}$ are written as $1,2,3,4$ respectively. The quantities $\bar{A}, \bar{B}, \bar{C}, \bar{D}$ are (see App. B)

$$
\begin{aligned}
\bar{A}\left(t, t^{\prime}, \mathbf{R}, \mathbf{R}^{\prime}\right) & \equiv \sum_{i} a_{i}(t) a_{i}\left(t^{\prime}\right) \delta\left(\mathbf{r}_{i}(t)-\mathbf{R}\right) \delta\left(\mathbf{r}_{i}\left(t^{\prime}\right)-\mathbf{R}^{\prime}\right) \\
\bar{B}\left(t, t^{\prime}, \mathbf{R}, \mathbf{R}^{\prime}\right) & \equiv \sum_{i} \delta\left(\mathbf{r}_{i}(t)-\mathbf{R}\right) \delta\left(\mathbf{r}_{i}\left(t^{\prime}\right)-\mathbf{R}^{\prime}\right) \\
\bar{C}\left(t, t^{\prime}, \mathbf{R}, \mathbf{R}^{\prime}\right) & \equiv \sum_{i} a_{i}(t) \delta\left(\mathbf{r}_{i}(t)-\mathbf{R}\right) \delta\left(\mathbf{r}_{i}\left(t^{\prime}\right)-\mathbf{R}^{\prime}\right) \\
\bar{D}\left(t, t^{\prime}, \mathbf{R}, \mathbf{R}^{\prime}\right) & \equiv \sum_{i} a_{i}\left(t^{\prime}\right) \delta\left(\mathbf{r}_{i}(t)-\mathbf{R}\right) \delta\left(\mathbf{r}_{i}\left(t^{\prime}\right)-\mathbf{R}^{\prime}\right)
\end{aligned}
$$


Note that $\bar{B}$ is the dynamic version of the order parameter used in Ref. [4]. In spin glass dynamics it is also found that the static Edwards-Anderson order parameter should be generalized to a time-dependent order parameter whose long time limit gives the static order parameter [8,43]. Similarly, the static order parameter used in Ref. [4] can be defined as the long time (equilibrium) limit of this dynamic order parameter $\bar{B}$. The physical meaning of the static order parameter is explained in Ref. [4]. We will explain later the physical meaning of the dynamical order parameter $\bar{B}$.

A little reflection tells us that, firstly, the terms $\langle\bar{A}\rangle \bar{B}$ and $\langle\bar{C}\rangle \bar{B}$ will not appear in the final effective equation of motion because they are related to the Jacobian when writing the functional integral representation for the effective equation of motion. As explained earlier, the Jacobian does not play an important role, as far as the effective equation of motion is concerned. Hence, we will also ignore these terms from now on. Secondly, the term $\langle\bar{B}\rangle \bar{A}$ is related to the stochastic part of the effective equation of motion because it contains two auxiliary fields outside of the self-consistent average bracket \langle\rangle . Finally, because of having only one auxiliary field outside of the self-consistent average bracket, the terms $\langle\bar{C}\rangle \bar{D}$ and $\langle\bar{B}\rangle \bar{C}$ are associated with the deterministic part.

We then take the average over $\boldsymbol{\theta}_{0}$ and $\boldsymbol{\xi}_{0}$. Because of their Gaussian character, these averages are easy to carry out. Note that these averages give

$$
\left\langle\exp \int d t \sum_{i} i \hat{\mathbf{r}}_{i}(t) \cdot\left(-\boldsymbol{\theta}_{0 i}(t)\right\rangle_{\theta_{0}}=\exp \left[\frac{1}{2} \int d t d t^{\prime} \sum_{i} \sum_{j} i \hat{\mathbf{r}}_{i}(t) \cdot\left\langle\boldsymbol{\theta}_{0 i}(t) \boldsymbol{\theta}_{0 j}\left(t^{\prime}\right)\right\rangle \cdot \dot{i} \hat{\mathbf{r}}_{j}\left(t^{\prime}\right)\right]\right.
$$

and

$$
\begin{aligned}
\left\langle\exp \int d t\right. & \left.\sum_{i} i \hat{\mathbf{r}}_{i}(t) \cdot\left[-g_{0} \int_{\mathbf{k}} \int_{-\infty}^{t} d t^{\prime} e^{-\nu_{0} k^{2}\left(t-t^{\prime}\right)} \mathbf{P}^{T}(\hat{\mathbf{k}}) \cdot \boldsymbol{\xi}_{0}\left(\mathbf{k}, t^{\prime}\right) e^{i \mathbf{k} \cdot \mathbf{r}_{i}(t)}\right]\right\rangle_{\xi_{0}} \\
= & \exp \left\{\sum _ { i } \sum _ { j } \int d t d t ^ { \prime } i \hat { \mathbf { r } } _ { i } ( t ) \cdot \left[\frac{g_{0}^{2}}{2} \int d \bar{t} d \bar{t}^{\prime} \int_{\mathbf{k}} \int_{\mathbf{k}^{\prime}} \theta(t-\bar{t}) \theta\left(t^{\prime}-\bar{t}^{\prime}\right) e^{-\nu_{0} k^{2}(t-\bar{t})} e^{-\nu_{0} k^{\prime 2}\left(t^{\prime}-\bar{t}^{\prime}\right)}\right.\right. \\
& \left.\left.\times e^{i \mathbf{k} \cdot \mathbf{r}_{i}(t)} e^{i \mathbf{k}^{\prime} \cdot \mathbf{r}_{j}\left(t^{\prime}\right)}\left\langle\boldsymbol{\xi}_{0}^{\perp}(\mathbf{k}, \bar{t}) \boldsymbol{\xi}_{0}^{\perp}\left(\mathbf{k}^{\prime}, \bar{t}^{\prime}\right)\right\rangle\right] \cdot i \hat{\mathbf{r}}_{j}\left(t^{\prime}\right)\right\}
\end{aligned}
$$

in $\left\langle\exp L_{0}\right\rangle_{\theta_{0} \xi_{0}}$ and contribute additional terms to the random part of the effective equation of motion, which is the term $\langle\bar{B}\rangle \bar{A}$ in Eq. (3.9). Bearing this in mind, we can easily write down the effective equation of motion. 


\section{Effective equation of motion and Markov approximation}

\section{Effective equation of motion}

It is a simple matter to read off the effective equation of motion after the average over Langevin noises is taken. We can explicitly write down the expression for $\langle\bar{B}\rangle \bar{A}$ in Eq. (3.9) and attribute terms involving tensor $\boldsymbol{\Gamma}_{i j}$ to renormalized noise $\boldsymbol{\xi}$ and the rest terms to renor-

malized noise $\boldsymbol{\theta}$. (See Eqs. (3.15) and (3.16) below.). Because the algebra is straightforward and slightly long, we will not write down the details here. The result is

$$
\frac{\partial}{\partial t} \mathbf{r}_{i}(t)=-\frac{1}{\zeta_{0}} \frac{\partial H_{0}}{\partial \mathbf{r}_{i}(t)}+g_{0} \mathcal{J}_{0}-\frac{2 B^{2}}{\zeta_{0}^{2}} \mathcal{J}_{1}-\frac{2 B_{0}}{\zeta_{0}} \mathcal{J}_{2}+\boldsymbol{\theta}_{i}(t)
$$

where

$$
\mathcal{J}_{0}=\int_{\mathbf{k}} e^{i \mathbf{k} \cdot \mathbf{r}} \int_{-\infty}^{t} d t^{\prime} e^{-\nu_{0} k^{2}\left(t-t^{\prime}\right)} \mathbf{P}^{T}(\hat{\mathbf{k}}) \cdot\left[\boldsymbol{\xi}_{0}\left(\mathbf{k}, t^{\prime}\right)-\frac{g_{0}}{\rho_{0}} \sum_{i=0}^{N-1} \frac{\partial H_{0}}{\partial \mathbf{r}_{i}\left(t^{\prime}\right)} e^{-i \mathbf{k} \cdot \mathbf{r}_{i}\left(t^{\prime}\right)}\right]
$$

is a vector field similar to the original solvent velocity field Eq. (2.5b). The renormalized Langevin noises are still Gaussian, with variances

$$
\left\langle\boldsymbol{\theta}_{i}(t) \boldsymbol{\theta}_{j}\left(t^{\prime}\right)\right\rangle=\left\langle\boldsymbol{\theta}_{0 i}(t) \boldsymbol{\theta}_{0 j}\left(t^{\prime}\right)\right\rangle+\frac{B^{2}}{2 \zeta_{0}^{2}} \mathcal{J}_{3}
$$

and

$$
\left\langle\boldsymbol{\xi}^{\perp}(\mathbf{k}, t) \boldsymbol{\xi}^{\perp}\left(\mathbf{k}^{\prime}, t^{\prime}\right)\right\rangle=\left\langle\boldsymbol{\xi}_{0}^{\perp}(\mathbf{k}, t) \boldsymbol{\xi}_{0}^{\perp}\left(\mathbf{k}^{\prime}, t^{\prime}\right)\right\rangle+\frac{B^{2} g_{0}^{2}}{2 \rho_{0}^{2}} \mathcal{J}_{4}
$$

In these equations, for convenience, we have defined vectorial quantities $\mathcal{J}_{1}$ and $\mathcal{J}_{2}$ and tensorial quantities $\mathcal{J}_{3}$ and $\mathcal{J}_{4}$. Their expressions are

$$
\begin{aligned}
\mathcal{J}_{1}= & \frac{1}{2} \int d t^{\prime} d 1 d 2\left\langle\bar{D}\left(t, t^{\prime}, 1,2\right)\right\rangle \boldsymbol{\nabla}_{i}(t) U\left(\mathbf{r}_{i}(t)-1\right) U\left(\mathbf{r}_{i}\left(t^{\prime}\right)-2\right) \\
+ & \frac{1}{2} \sum_{i} \int d t^{\prime} d \bar{t} d 1 d 2\left\langle\bar{D}\left(t^{\prime}, \bar{t}, 1,2\right)\right\rangle \theta\left(t-t^{\prime}\right) \boldsymbol{\Gamma}_{i j}\left(t, t^{\prime}\right) \cdot \nabla_{j}\left(t^{\prime}\right) U\left(\mathbf{r}_{j}\left(t^{\prime}\right)-1\right) U\left(\mathbf{r}_{j}(\bar{t})-2\right) \\
& \mathcal{J}_{2}=\frac{1}{2} \int d 1 d 2\langle\bar{B}(t, t, 1,2)\rangle \boldsymbol{\nabla}_{i}(t) U\left(\mathbf{r}_{i}(t)-1\right) \\
& +\frac{1}{2} \sum_{i} \int d t^{\prime} d 1 d 2\left\langle\bar{B}\left(t^{\prime}, t^{\prime}, 1,2\right)\right\rangle \theta\left(t-t^{\prime}\right) \boldsymbol{\Gamma}_{i j}\left(t, t^{\prime}\right) \cdot \nabla_{j}\left(t^{\prime}\right) U\left(\mathbf{r}_{j}\left(t^{\prime}\right)-1\right)
\end{aligned}
$$




$$
\begin{aligned}
\mathcal{J}_{3}= & \delta_{i j} \int d 1 d 2\left\langle\bar{B}\left(t, t^{\prime}, 1,2\right)\right\rangle \boldsymbol{\nabla}_{i}(t) \boldsymbol{\nabla}_{i}\left(t^{\prime}\right) U\left(\mathbf{r}_{i}(t)-1\right) U\left(\mathbf{r}_{i}\left(t^{\prime}\right)-2\right) \\
& +2 \int d \bar{t} d 1 d 2\left\langle\bar{B}\left(\bar{t}, t^{\prime}, 1,2\right)\right\rangle \theta(t-\bar{t}) \boldsymbol{\Gamma}_{i j}(t, \bar{t}) \cdot \boldsymbol{\nabla}_{j}(\bar{t}) \boldsymbol{\nabla}_{j}\left(t^{\prime}\right) U\left(\mathbf{r}_{j}(\bar{t})-1\right) U\left(\mathbf{r}_{j}\left(t^{\prime}\right)-2\right) \\
= & \delta_{i j} \int d 1 d 2\left\langle\bar{B}\left(t, t^{\prime}, 1,2\right)\right\rangle \boldsymbol{\nabla}_{i}(t) \boldsymbol{\nabla}_{i}\left(t^{\prime}\right) U\left(\mathbf{r}_{i}(t)-1\right) U\left(\mathbf{r}_{i}\left(t^{\prime}\right)-2\right) \\
+ & 2 \int d \bar{t} d 1 d 2\langle\bar{B}(t, \bar{t}, 1,2)\rangle \theta\left(t^{\prime}-\bar{t}\right) \boldsymbol{\nabla}_{i}(t) \boldsymbol{\Gamma}_{j i}\left(t^{\prime}, \bar{t}\right) \cdot \boldsymbol{\nabla}_{i}(\bar{t}) U\left(\mathbf{r}_{i}(\bar{t})-1\right) U\left(\mathbf{r}_{i}(\bar{t})-2\right) \\
& \mathcal{J}_{4}=\sum_{i} \int d 1 d 2\left\langle\bar{B}\left(t, t^{\prime}, 1,2\right)\right\rangle e^{-i \mathbf{k} \cdot \mathbf{r}_{j}(t)} e^{-i \mathbf{k}^{\prime} \cdot \mathbf{r}_{j}\left(t^{\prime}\right)} \\
& \times \mathbf{P}^{T}(\hat{\mathbf{k}}) \cdot \nabla_{j}(t) \mathbf{P}^{T}\left(\hat{\mathbf{k}}^{\prime}\right) \cdot \boldsymbol{\nabla}_{j}\left(t^{\prime}\right) U\left(\mathbf{r}_{j}(t)-1\right) U\left(\mathbf{r}_{j}\left(t^{\prime}\right)-2\right)
\end{aligned}
$$

(We have suppressed all the possible dependence of these $\mathcal{J}$ 's on time, monomer index, and so on.)

Note that, although Eq. (3.14) is essentially the same as Eq. (2.5b), it would be incorrect to write a separate Navier-Stokes equation, similar to Eq. (2.40), for $\mathcal{J}_{0}$, in which the full Hamiltonian is replaced by $H_{0}$, and claim that when the average over the quenched randomness is performed the effective equation of motion for solvent velocity is not changed. Recall that we have eliminated the solvent velocity field before the average is performed. In priciple, one can explicitly introduce an auxiliary field for the solvent velocity and perform the average over the quenched randomness to find effective equations of motion for both polymer conformation and solvent velocity. The effective equation of motion for solvent velocity field is presumably different from the original Navier-Stokes equation, though we expect that we would get the same equation as Eq. (3.13) once the velocity field is projected out. As we have mentioned in Sec. II, our concern here is the equation of motion of the chain. Therefore, we will make no attempt on deriving an effective Oono-Freed kinetic equation for solvent velocity field.

Following the conventional interpretation of MSR formalism [7, 33], time-correlation functions of dynamical variables $\left\{\mathbf{r}_{i}\right\}$ and their auxiliary fields $\left\{\hat{\mathbf{r}}_{i}\right\}$ are response functions of the system to the perturbation associated with $\left\{\hat{\mathbf{r}}_{i}\right\}$. Causality requires that these response functions should be vanishing if any of the time variables of the auxiliary fields $\left\{\hat{\mathbf{r}}_{i}\right\}$ is larger than all the time variables of the dynamical variables $\left\{\mathbf{r}_{i}\right\}$. Imposing this causality require- 
ment upon Eqs. (3.10) we can show that causality is also respected in the effective equation of motion, Eq. (3.13).

\section{Markov approximation}

The essential ingredients of the proof given in App. A are (1) elimination of solvent velocity field, and (2) Markov approximation. Physically, (1) means average over the solvent velocity and (2) means that the characteristic time scale for solvent dynamics is much shorter than the characteristic time scale for the motion of the chain.

We adopt the same spirit of App. A. As the velocity field has been eliminated, we now

apply the Markov approximation to further simplify the effective equation of motion. The Markov approximation is

$$
e^{-\nu_{0} k^{2}\left(t-t^{\prime}\right)} \rightarrow \frac{2}{\nu_{0} k^{2}} \delta\left(t-t^{\prime}\right)
$$

Within this approximation, the Oseen tensor

$$
\mathbf{T}\left(\mathbf{r}, \mathbf{r}^{\prime}\right) \equiv \int_{\mathbf{k}} \frac{1}{\eta_{0} k^{2}} \mathbf{P}^{T}(\hat{\mathbf{k}}) e^{i \mathbf{k} \cdot\left(\mathbf{r}-\mathbf{r}^{\prime}\right)}
$$

appears, for example,

$$
\boldsymbol{\Gamma}_{i j}\left(t, t^{\prime}\right)=2 g_{0}^{2} \zeta_{0} \delta\left(t-t^{\prime}\right) \mathbf{T}\left(\mathbf{r}_{i}(t), \mathbf{r}_{j}(t)\right)
$$

Using Markov approximation we can largely simplify the effective equation of motion.

Since we seek the result only to the lowest nontrivial order, that is, to the lowest order in $B, g_{0}$, and $B_{0}$, we can simplfy the expressions of $\mathcal{J}$ 's by dropping all the higher order terms. For convenience, we define

$$
\begin{gathered}
\bar{\rho}(\mathbf{R}, t) \equiv\left\langle\sum_{i} \delta\left(\mathbf{r}_{i}(t)-\mathbf{R}\right)\right\rangle \\
\boldsymbol{G}\left(t, t^{\prime}, 1,2\right) \equiv\left\langle\sum_{i} \delta\left(\mathbf{r}_{i}(t)-1\right) i \hat{\mathbf{r}}_{i}\left(t^{\prime}\right) \delta\left(\mathbf{r}_{i}\left(t^{\prime}\right)-2\right)\right\rangle
\end{gathered}
$$




$$
C\left(t, t^{\prime}, 1,2\right) \equiv\left\langle\sum_{i} \delta\left(\mathbf{r}_{i}(t)-1\right) \delta\left(\mathbf{r}_{i}\left(t^{\prime}\right)-2\right)\right\rangle
$$

where the bracket is the mean-field average defined by Eq. (B13) in App. B. The mean-field kinetic equation, Eq. (3.13), then reduces to

$$
\frac{\partial}{\partial t} \mathbf{r}_{i}(t)=-\frac{1}{\zeta_{0}} \frac{\partial H_{e f f}}{\partial \mathbf{r}_{i}(t)}-g_{0}^{2} \sum_{j} \mathbf{T}\left(\mathbf{r}_{i}, \mathbf{r}_{j}\right) \cdot \frac{\partial H_{e f f}}{\partial \mathbf{r}_{j}(t)}+\boldsymbol{\mu}_{i}(t)
$$

where the new Langevin noise $\boldsymbol{\mu}$ is Gaussian with variance

$$
\begin{aligned}
\left\langle\boldsymbol{\mu}_{i}(t) \boldsymbol{\mu}_{j}\left(t^{\prime}\right)\right\rangle= & \frac{2}{\zeta_{0}} \delta\left(t-t^{\prime}\right) \delta_{i j} \mathbf{1}+2 g_{0}^{2} \delta\left(t-t^{\prime}\right) \mathbf{T}\left(\mathbf{r}_{i}(t), \mathbf{R}_{j}(t)\right) \\
& +\frac{B^{2}}{2 \zeta_{0}^{2}} \delta_{i j} \int d 1 d 2 C\left(t, t^{\prime}, 1,2\right) \frac{\partial U\left(\mathbf{r}_{i}(t)-1\right)}{\partial \mathbf{r}_{i}(t)} \frac{\partial U\left(\mathbf{r}_{i}\left(t^{\prime}\right)-2\right)}{\partial \mathbf{r}_{i}\left(t^{\prime}\right)}
\end{aligned}
$$

The effective Hamiltonian $H_{\text {eff }}$ becomes

$$
\begin{aligned}
H_{e f f}=H_{0}+ & B_{0} \sum_{i} \int d 1 \bar{\rho}(\mathbf{r}, t) U\left(\mathbf{r}_{i}(t)-1\right) \\
& -\frac{B^{2}}{2 \zeta_{0}} \sum_{i} \int_{\infty}^{t^{-}} d t^{\prime} \int d 1 d 2 \boldsymbol{G}\left(t, t^{\prime}, 1,2\right) \cdot \frac{\partial U\left(\mathbf{r}_{i}\left(t^{\prime}\right)-2\right)}{\partial \mathbf{r}_{i}\left(t^{\prime}\right)} U\left(\mathbf{r}_{i}(t)-1\right)
\end{aligned}
$$

where $t^{-} \equiv t-\epsilon, \epsilon$ being an infinitesimal positive number.

These equations have the form of the nonlinear generalized Langevin equation 44,45. However, the reason for the emergence of this nonlinear generalized Langevin equation is different from that in Refs. 44,45]. It is from the elimination of heterogeneity of the original system, rather than from the elimination of the degrees of freedom of surrounding "bath" molecules. Here the elimination of the surrounding "bath" molecules does not cause any memory effect because Markov approximation is used.

In generalized hydrodynamics one can define frequency and wavelength dependent transport coefficients [46]. Similarly, in Eq. (3.29), the term containing heterogeneity $B$ can be seen as giving a time-dependent excluded volume interaction. Alternatively, since this term is non-local in time, one can say that the heterogeneity introduces an excluded volume interaction along the world line, in addition to the usual excluded volume interaction $B_{0}$ along the chain contour. 
Inspecting these equations, we find that they are similar, albeit more complicated, to the effective kinetic equation obtained in spin glass dynamics [8.9]. In these works a memory function also appears in mean-field limit. In principle, one can expand the monomermonomer interaction $U$ in terms of dynamical variables $\mathbf{r}_{i}(t)$. This expansion will lead to a generalization of the $p$-spin-interaction spin-glass dynamics discussed in Ref. [9]. However, since one will get infinite $p$-spin-interaction terms, this approach does not seem to be practically feasible.

We can now take the known result for homopolymer as a reference. When we set the chain heterogeneity $B$ zero, Eq. (3.27) is expected to reduce to the same equation obtained from eliminating the solvent velocity field in the Oono-Freed kinetic equation (See App. A),

$$
\begin{gathered}
\frac{\partial}{\partial t} \mathbf{r}_{i}(t)=-\frac{1}{\zeta_{0}} \frac{\partial H_{E}}{\partial \mathbf{r}_{i}(t)}-g_{0}^{2} \sum_{j} \mathbf{T}\left(\mathbf{r}_{i}(t), \mathbf{r}_{j}(t)\right) \cdot \frac{\partial H_{E}}{\partial \mathbf{r}_{j}(t)}+\boldsymbol{\mu}_{i}^{E}(t) \\
\left\langle\boldsymbol{\mu}_{i}^{E}(t) \boldsymbol{\mu}_{j}^{E}\left(t^{\prime}\right)\right\rangle=\frac{2}{\zeta_{0}} \delta\left(t-t^{\prime}\right) \delta_{i j} \mathbf{1}+2 g_{0}^{2} \delta\left(t-t^{\prime}\right) \mathbf{T}\left(\mathbf{r}_{i}(t), \mathbf{r}_{j}(t)\right)
\end{gathered}
$$

where $H_{E}$ is the Edwards Hamiltonian (2.3). This equation reveals that, the lowest order approximation of $\bar{\rho}(\mathbf{R}, t)$ must be the usual monomer density. Any higher order correction, after multiplied by $B_{0}$, must be first or higher order in $B_{0}, g_{0}^{2} / \eta_{0}$, or $B^{2} / \zeta_{0}$. To the order of our calculation, these corrections can be dropped. Therefore, we can replace $\bar{\rho}(\mathbf{R}, t)$ by its corresponding un-bracketed quantity:

$$
\bar{\rho}(\mathbf{R}, t) \equiv \sum_{i} \delta\left(\mathbf{r}_{i}(t)-\mathbf{R}\right)
$$

Taking into account the symmetry of monomer indices, which should be restored when the self-consistent bracket is removed, the effective Hamiltonian (3.29) becomes

$$
\begin{aligned}
H_{e f f}=H_{0}+ & \frac{B_{0}}{2} \sum_{i j} U\left(\mathbf{r}_{i}(t)-\mathbf{r}_{j}(t)\right) \\
& -\frac{B^{2}}{2 \zeta_{0}} \sum_{i} \int_{\infty}^{t^{-}} d t^{\prime} \int d 1 d 2 \boldsymbol{G}\left(t, t^{\prime}, 1,2\right) \cdot \frac{\partial U\left(\mathbf{r}_{i}\left(t^{\prime}\right)-2\right)}{\partial \mathbf{r}_{i}\left(t^{\prime}\right)} U\left(\mathbf{r}_{i}(t)-1\right)
\end{aligned}
$$

Since there is no corresponding known result for $B_{0}=0$ and $B \neq 0$ (a heteropolymer chain in theta solution), we cannot find a lowest order approximation for $\boldsymbol{G}$ using the same method. 
The quantity $\boldsymbol{G}\left(t, t^{\prime}, \mathbf{R}_{1}, \mathbf{R}_{2}\right)$ in Eq. (3.33) gives the response field of the chain at time $t$, when the chain conformation is $\delta\left(\mathbf{r}_{i}(t)-\mathbf{R}_{1}\right)$, to a perturbation, introduced by $\hat{\mathbf{r}}_{i}\left(t^{\prime}\right)$ at time $t^{\prime}$, when the chain conformation was $\delta\left(\mathbf{r}_{i}\left(t^{\prime}\right)-\mathbf{R}_{2}\right)$. (The perturbation can be explicitly introduced in the kinetic equation, in the same way as the perturbative magnetic field used in [8,9]. For simplicity it is not included here.) Causality again is respected and the integral of $t^{\prime}$ in the effective Hamiltonian is limited by current time $t$.

The quantity $C\left(t, t^{\prime}, \mathbf{R}_{1}, \mathbf{R}_{2}\right)$ in the correlation function of the renormalized noise $\boldsymbol{\mu}_{i}$, Eq. (3.28), gives the correlation function of chain conformations, between conformation $\delta\left(\mathbf{r}_{i}(t)-\mathbf{R}_{1}\right)$ at time $t$ and $\delta\left(\mathbf{r}_{i}\left(t^{\prime}\right)-\mathbf{R}_{2}\right)$ at time $t^{\prime}$. This is a natural dynamical generalization of the static order parameter proposed in Ref. [4].

Note that the effective Hamiltonian is a time-dependent Hamiltonian. The memory function $\boldsymbol{G}\left(t, t^{\prime}\right)$ makes the chain conformation at time $t$ depend on all the past chain conformations. This memory term makes the kinetic equation non-Markovian, and writing a Fokker-Planck equation for it impossible. It is well known that a non-Markovian process can be made Markovian by introducing new degrees of freedom (e.g. the exmaple in Ref. [47]). However, in our case, this would be equivalent to going back to the original OonoFreed kinetic equations where the process was Markovian, because what has been done is exactly elimination of some degrees of freedom (heterogeneity and solvent) from the original Markovian process. Of course, a Fokker-Planck-like equation can be written for Eqs. (3.27) and (3.33), but this would not give a closed equation. Instead, it gives an equation with infinite terms (e.g. a Kramers-Moyal expansion) [31].

On the other hand, a Fokker-Planck equation with memory effect has been developed by Zwanzig [48]. It is also shown in Ref. [48] that, after certain approximations, the memory Fokker-Planck equation can be transformed into a generalized Langevin equation. This seems to allow us to transform the generalized Langevin equation obtained here back to a memory Fokker-Planck equation. However, it is not clear how to perform this backward transformation.

These considerations make it clear that trying to write down a generalized (memory) 
Kirkwood diffusion equation for the mean field equation of motion Eq. (3.27) would be very difficult, if not impossible. Fortunately, although the relaxation spectrum is calculated in Ref. [17] by perturbatively solving the Kirkwood diffusion equation, one can also derive the relaxation times directly from the kinetic equation. As it is clear from the above analysis, we should not try to find the corresponding generalized Kirkwood diffusion equation but rather derive the relaxation spectrum directly from the kinetic equation. This is done in the next section.

\section{MODE RELAXATION SPECTRUM}

\section{A. Transition to continuous model}

For convenience, we now rewrite the effective equation of motion in terms of continuous chain notation. We will use the standard notation [12,19]: $\tau$ being the contour length measured along the chain; $\mathbf{c}(\tau, t)$ the position of the point at $\tau$. A short distance cut-off $a$ will be implicitly assumed in the following equations so that $\left|\tau-\tau^{\prime}\right|>a$ for all double integrals over $\tau$ and $\tau^{\prime}$. The upper limit of contour length can be $N-1$, according to Eq. (2.1), or $N$, because $N$ has been taken to be very large in mean field approximation. We will use $N$ as the upper limit of contour length so that one can readily compare our result to the result of Ref. [17.

The effective equation of motion in continuous chain notation is

$$
\frac{\partial}{\partial t} \mathbf{c}(\tau, t)=-\frac{1}{\zeta_{0}} \frac{\delta H_{e f f}}{\delta \mathbf{c}(\tau, t)}-g_{0}^{2} \int d \tau^{\prime} \mathbf{T}\left(\mathbf{c}(\tau, t)-\mathbf{c}\left(\tau^{\prime}, t\right)\right) \cdot \frac{\delta H_{e f f}}{\delta \mathbf{c}\left(\tau^{\prime}, t\right)}+\boldsymbol{\mu}(\tau, t),
$$

where

$$
\begin{aligned}
&\left\langle\boldsymbol{\mu}(\tau, t) \boldsymbol{\mu}\left(\tau^{\prime}, t^{\prime}\right)\right\rangle= \frac{2}{\zeta_{0}} \mathbf{1} \delta\left(t-t^{\prime}\right) \delta\left(\tau-\tau^{\prime}\right)+2 g_{0}^{2} \delta\left(t-t^{\prime}\right) \mathbf{T}\left(\mathbf{c}(\tau, t)-\mathbf{c}\left(\tau^{\prime}, t^{\prime}\right)\right) \\
&+\frac{B^{2}}{2 \zeta_{0}^{2}} \delta\left(\tau-\tau^{\prime}\right) \int d 1 d 2 C\left(t, t^{\prime}, 1,2\right) \frac{\delta U(\mathbf{c}(\tau, t)-1)}{\mathbf{c}(\tau, t)} \frac{\delta U\left(\mathbf{c}\left(\tau^{\prime}, t^{\prime}\right)-2\right)}{\mathbf{c}\left(\tau^{\prime}, t^{\prime}\right)} \\
& H_{e f f}=H_{E}-\frac{B^{2}}{2 \zeta_{0}} \int d \tau \int_{-\infty}^{t^{-}} d t^{\prime} \int d 1 d 2 \boldsymbol{G}\left(t, t^{\prime}, 1,2\right) \cdot \frac{\delta U\left(\mathbf{c}\left(\tau, t^{\prime}\right)-2\right)}{\delta \mathbf{c}\left(\tau, t^{\prime}\right)} U(\mathbf{c}(\tau, t)-1)
\end{aligned}
$$




$$
\begin{gathered}
H_{E}=\frac{1}{2} \int d \tau\left|\frac{\partial}{\partial \tau} \mathbf{c}(\tau, t)\right|^{2}+\frac{B_{0}}{2} \int d \tau d \tau^{\prime} U\left(\mathbf{c}(\tau, t)-\mathbf{c}\left(\tau^{\prime}, t\right)\right) \\
\boldsymbol{G}\left(t, t^{\prime}, 1,2\right)=\int d \tau\left\langle\delta(\mathbf{c}(\tau, t)-1) i \hat{\mathbf{c}}\left(\tau, t^{\prime}\right) \delta\left(\mathbf{c}\left(\tau^{\prime}, t\right)-2\right)\right\rangle \\
C\left(t, t^{\prime}, 1,2\right)=\int d \tau\left\langle\delta(\mathbf{c}(\tau, t)-1) \delta\left(\mathbf{c}\left(\tau^{\prime}, t\right)-2\right)\right\rangle \\
\mathbf{T}\left(\mathbf{c}(\tau, t)-\mathbf{c}\left(\tau^{\prime}, t\right)\right)=\int_{\mathbf{k}} \frac{1}{\eta_{0} k^{2}} \mathbf{P}^{T}(\hat{\mathbf{k}}) e^{i \mathbf{k} \cdot\left(\mathbf{c}(\tau, t)-\mathbf{c}\left(\tau^{\prime}, t\right)\right)}
\end{gathered}
$$

From now on the potential $U\left(\mathbf{c}(\tau, t)-\mathbf{c}\left(\tau^{\prime}, t\right)\right)$ will be taken as a hard-core repulsive interaction $\delta\left(\mathbf{c}(\tau, t)-\mathbf{c}\left(\tau^{\prime}, t\right)\right)$ so that we can compare our result with Ref. [17]. For a general potential the corresponding equations can be easily written down.

Eq. (4.1) essentially describes a non-equilibrium process. In spin glass dynamics, as mentioned above, a similar equation can be obtained through the same mean field approximation [8,9]. In Refs. [8,9], it is assumed that the process is stationary. While the newly developed out-of-equilibrium dynamics [49] is potentially able to address non-stationary dynamics, we will assume that in our case the process is stationary. This assumption gives

$$
\begin{aligned}
& \boldsymbol{G}\left(t, t^{\prime}, 1,2\right)=\boldsymbol{G}\left(t-t^{\prime}, 1,2\right) \\
& C\left(t, t^{\prime}, 1,2\right)=C\left(t-t^{\prime}, 1,2\right)
\end{aligned}
$$

\section{B. Perturbation calculation}

The conventional method used to determine the spectrum of mode relaxation, especially the longest relaxation time, is to find the eigenvalues and eigenfunctions for the FokkerPlanck operator [17. This approach is useful as long as a Fokker-Planck equation exists. However, as we have analyzed in the previous section, this is not the case here.

Nevertheless, since what we want to find is the longest relaxation time, we can simply use perturbation method to find out how the kinetic coefficient is renormalized, with the same 
spirit of Refs. [50,51]. In principle, it is possible to develop a diagrammatic method, similar to the diagrammatics developed in Ref. [52], to systematically calculate desired quantities. However, this would not make much sense because Eqs. (4.1) is valid only to the lowest nontrivial order. A straightforward perturbation calculation will be sufficient.

Introducing Rouse coordinates $\boldsymbol{\xi}(p, t)$,

$$
\boldsymbol{\xi}(p, t) \equiv \sqrt{\frac{2}{N}} \int d \tau \mathbf{c}(\tau, t) \cos \frac{p \pi \tau}{N}
$$

and its Fourier transform

$$
\boldsymbol{\xi}(p, \omega) \equiv \int d t \boldsymbol{\xi}(p, t) e^{i \omega t}
$$

the equation of motion becomes

$$
\begin{aligned}
& G_{0}^{-1}(p, \omega) \boldsymbol{\xi}(p, \omega)=-\frac{B_{0}}{\zeta_{0}} \sqrt{\frac{2}{N}} \int d \tau d \tau^{\prime} d t \int_{\mathbf{k}} e^{i \omega t} i \mathbf{k} \cos \frac{p \pi \tau}{N} \\
& \times \exp \left[i \sqrt{\frac{2}{N}} \mathbf{k} \cdot \sum_{p^{\prime}} \int_{\omega^{\prime}} e^{-i \omega t^{\prime}} \boldsymbol{\xi}\left(p^{\prime}, \omega^{\prime}\right) Q_{p^{\prime}}\left(\tau, \tau^{\prime}\right)\right] \\
& -g_{0}^{2} \frac{2}{N} \int d \tau d \tau^{\prime} d t \int_{\mathbf{k}} \int_{\omega} \sum_{p^{\prime}} e^{i \omega t}\left(\frac{p^{\prime} \pi}{N}\right)^{2} \frac{1}{\eta_{0} k^{2}} \cos \frac{p \pi \tau}{N} \cos \frac{p^{\prime} \pi \tau^{\prime}}{N} \\
& \times \exp \left[i \sqrt{\frac{2}{N}} \mathbf{k} \cdot \sum_{p^{\prime \prime}} \int_{\omega^{\prime \prime}} e^{-i \omega^{\prime \prime} t} \boldsymbol{\xi}\left(p^{\prime \prime}, \omega^{\prime \prime}\right) Q_{p^{\prime \prime}}\left(\tau, \tau^{\prime}\right)\right] \mathbf{P}^{T}(\hat{\mathbf{k}}) \cdot \boldsymbol{\xi}\left(p^{\prime}, \omega^{\prime}\right) e^{-i \omega^{\prime} t} \\
& +\frac{B^{2}}{2 \zeta_{0}^{2}} \sqrt{\frac{2}{N}} \int d t d \tau d 1 d 2 \int_{-\infty}^{t^{-}} d t^{\prime} \int_{\mathbf{k}} \int_{\mathbf{k}^{\prime}} e^{i \omega t} \boldsymbol{G}\left(t, t^{\prime}, 1,2\right) \cdot i \mathbf{k}^{\prime} i \mathbf{k} \cos \frac{p \pi \tau}{N} \\
& \times e^{-i \mathbf{k}^{\prime} \cdot \mathbf{R}_{2}} \exp \left[i \sqrt{\frac{2}{N}} \mathbf{k}^{\prime} \cdot \sum_{p^{\prime \prime}} \int_{\omega^{\prime \prime}} \boldsymbol{\xi}\left(p^{\prime \prime}, \omega^{\prime \prime}\right) e^{-i \omega^{\prime \prime} t^{\prime}} \cos \frac{p^{\prime \prime} \pi \tau}{N}\right] \\
& \times e^{-i \mathbf{k} \cdot \mathbf{R}_{1}} \exp \left[i \sqrt{\frac{2}{N}} \mathbf{k} \cdot \sum_{p^{\prime}} \int_{\omega^{\prime}} \boldsymbol{\xi}\left(p^{\prime}, \omega^{\prime}\right) e^{-i \omega^{\prime} t} \cos \frac{p^{\prime} \pi \tau}{N}\right] \\
& +\boldsymbol{\mu}(p, \omega),
\end{aligned}
$$

and

$$
\begin{aligned}
\left\langle\boldsymbol{\mu}(p, \omega) \boldsymbol{\mu}\left(p^{\prime}, \omega^{\prime}\right)\right\rangle= & \frac{2}{N} \frac{2}{\zeta_{0}} \mathbf{1} \int d t d \tau e^{i\left(\omega+\omega^{\prime}\right) t} \cos \frac{p \pi \tau}{N} \cos \frac{p^{\prime} \pi \tau}{N} \\
& +\frac{2}{N} 2 g_{0}^{2} \int d t d \tau d \tau^{\prime} e^{i\left(\omega+\omega^{\prime}\right) t} \cos \frac{p \pi \tau}{N} \cos \frac{p^{\prime} \pi \tau^{\prime}}{N} \mathbf{T}\left(\mathbf{c}(\tau, t)-\mathbf{c}\left(\tau^{\prime}, t\right)\right) \\
& +\frac{2}{N} \frac{B^{2}}{2 \zeta_{0}^{2}} \int d t d \tau d t^{\prime} e^{i \omega t} e^{i \omega^{\prime} t^{\prime}} \cos \frac{p \pi \tau}{N} \cos \frac{p^{\prime} \pi \tau}{N} \int d 1 d 2 C\left(t, t^{\prime}, 1,2\right)
\end{aligned}
$$




$$
\times \frac{\delta \delta(\mathbf{c}(\tau, t)-1)}{\delta \mathbf{c}(\tau, t)} \frac{\delta \delta\left(\mathbf{c}\left(\tau, t^{\prime}\right)-2\right)}{\delta \mathbf{c}\left(\tau, t^{\prime}\right)}
$$

where we have defined

$$
\begin{gathered}
\int_{\omega} \equiv \int \frac{d \omega}{2 \pi}, \\
G_{0}^{-1}(p, \omega) \equiv-i \omega+\Lambda_{p}, \quad \Lambda_{p} \equiv \frac{1}{\zeta_{0}}\left(\frac{p \pi}{N}\right)^{2}, \\
Q_{p}\left(\tau, \tau^{\prime}\right) \equiv \cos \frac{p \pi \tau}{N}-\cos \frac{p \pi \tau^{\prime}}{N} .
\end{gathered}
$$

Since we need to carry out calculations only to the lowest nontrivial order, we can iterate this equation once and drop all the higher order terms. The detailed calculation can be found in App. Q. It is shown in App. Q that the relaxation spectrum $\Lambda_{p}$ is renormalized by three terms at the lowest nontrivial order,

$$
\begin{aligned}
& \delta \Lambda_{p}^{(1)}=-\frac{B_{0}}{\zeta_{0}} \frac{2}{N} \int d \tau d \tau^{\prime} \int_{\mathbf{k}} \frac{k^{2}}{d} \cos \frac{p \pi \tau}{N} Q_{p}\left(\tau, \tau^{\prime}\right) e^{-k^{2} B_{N}\left(\tau, \tau^{\prime}\right)} \\
& \delta \Lambda_{p}^{(2)}=-\frac{g_{0}^{2}}{\eta_{0}} \frac{2}{N}\left(\frac{p \pi}{N}\right)^{2} \int d \tau d \tau^{\prime} \int_{\mathbf{k}} \frac{1-1 / d}{k^{2}} \cos \frac{p \pi \tau}{N} \cos \frac{p \pi \tau^{\prime}}{N} e^{-k^{2} B_{N}\left(\tau, \tau^{\prime}\right)} \\
& \delta \Lambda_{p}^{(3)}=-\frac{B^{2}}{2 \zeta_{0}^{2}} \frac{2}{N} \int d \tau \int_{\mathbf{k}} \cos \frac{p \pi \tau}{N} \cos \frac{p \pi \tau^{\prime}}{N} i[\tilde{\boldsymbol{G}}(\tau, 0, \mathbf{k})-\tilde{\boldsymbol{G}}(\tau,-\omega, \mathbf{k})] \cdot \mathbf{k} \frac{k^{2}}{d}
\end{aligned}
$$

Compared to the result obtained in Ref. [17], Eqs. (4.17) and (4.18), renormalizations due to excluded volume interaction and hydrodynamic interaction respectively, coincide with the results reported there. The additional renormalization due to the chain heterogeneity $B$ is given by $\delta \Lambda_{p}^{(3)}$. It is found in Ref. [17] that the renormalizations from excluded volume and hydrodynamic effects are independent of each other, at the lowest nontrivial order. Here we see that the addition of heterogeneity preserves this independence: All three effects are not coupled.

It has been shown that in spin glasses there exists a critical temperature below which the replica symmetric solution is not stable [3]. Below this transition temperature, there are infinite ground states so that the system displays non-ergodic behavior. This non-ergodic 
behavior is responsible for many characteristics of the spin glasses below the transition temperature [3]. It is proposed in Ref. [8] that, in an infinite system, the fluctuationdissipation theorem (FDT) should be violated below the spin-glass transition temperature $T_{c}$. Mathematically, the violation comes from the extra time-persistent term in the correlation and response functions. Physically, the breaking of FDT is caused by the non-ergodic behavior of the system. The response function contains not only the usual response obeying the FDT but also a time-persistent part corresponding to the crossing of the energy barriers between the ground states. Ref. [8] also shows that the breaking of FDT is needed in order to make the static mean-field solution of the Sherrington-Kirkpatrick (SK) model stable.

It is clear that this dynamical consequence (i.e. breaking of FDT) is closely related to the static property (many ground states) of the SK model of spin glasses. Ref. [4] shows that a freezing transition can occur in heteropolymers at which only few conformations (states) dominate in the partition function. One might expect some dynamic consequences, and in fact it was suggested indeed [18] that static freezing in heteropolymers is accompanied by that dynamic freezing. This assertion was made based on the phenomenological analysis which extends the REM to kinetics. The model used in [18 employs an unphysical assumption that two states connected by one kinetic step have statistically independent energies; it is this assumption which leads to the conclusion about the glass transition in this system [53]. Our microscopic analysis, Eq. (4.19) shows that, within the order of our calculations whether the FDT is broken or not, dynamically, there is no effect at all. This is because the $\tilde{\boldsymbol{G}}(\tau, 0, \mathbf{k})$ term exactly cancels the time-persistent part of the response field, if the temperature is below the transition temperature. Since the time-persistent part of response function is directly related to the degeneracy of the ground states, this result implies that this degeneracy is dynamically unobservable, at least to the lowest nontrivial order. This, in turn may mean that barriers between low-energy states are nonextensive (in $N$ ) in the studied model of heteroplymer.

One may argue that the violation of FDT happens only when the system is infinitely large, $N=\infty$, and, since only large $N$ is imposed in the derivation of the effective equation 
of motion, breaking of FDT does not happen and the above argument is not quite right. Nevertheless, what really matters is that the term $\tilde{\boldsymbol{G}}(\tau, 0, \mathbf{k})-\tilde{\boldsymbol{G}}(\tau,-\omega, \mathbf{k})$ makes all static effects at zero frequencies dynamically null, and corresponding static freezing phenomena may not have its have its dynamic counterpart in this system.

Carefully checking the calculation that leads to the term $\tilde{\boldsymbol{G}}(\tau, 0, \mathbf{k})-\tilde{\boldsymbol{G}}(\tau,-\omega, \mathbf{k})$ in Eq. (4.19), we see that it is rooted in the two exponential functions in the $B$-dependent term in Eq. (4.12). This makes it clear that the chain flexibility is responsible for the dynamically null result. Physically, this is a plausible result. The quenched randomness in Eq. (2.1) is quenched along the chain, but not in the space in which the heteropolymer is embedded. As we do not expect a dilute liquid mixture of magnetic and non-magnetic particles displaying any "frozen in" behavior observed in its solid mixture counterpart, e.g. spin glasses, we do not expect a full spin-glass-like behavior in heteropolymers because the randomness is not fully quenched in space. We probably can say that the correspondence between spin glasses and heteropolymers is statically more complete than dynamically. The analogy between spin glasses and biopolymers therefore has to be used with some care, especially in building ad hoc phenomenological models not supported by microscopic analysis.

\section{ON THE ANALOGY BETWEEN SPIN GLASS AND HETEROPOLYMER}

It is well known that there exists an elegant correspondence between magnetic system and self-avoiding random walk, e.g. a polymer chain with excluded volume effect. This correspondence can be shown perturbatively and analytically [20,54,55. From the point of view of this complete correspondence, it seems that the analogy between spin glass and heteropolymer should be also complete. However, according the result obtain in the prvious section, one has to be careful when using this analogy. Therefore, it is necessary to think more about this analogy in the context of the correspondence.

There are different ways to establish the correspondence between magnetic system and self-avoiding random walk [20]. Here we will follow the analytical proof given in Ref. [55]. 
Using the result obtained in Ref. [55] we know that the model considered here corresponds to an Ising model with non-random nearest neighbor interaction and random four-spin coupling. More precisely, Eqs. (2.1) can be obtained from the $n=0$ limit of the following soft-spin Hamiltonian,

$$
H=\sum_{\alpha}^{n} \sum_{i j}^{N} J_{i j} \sigma_{i \alpha} \sigma_{j \alpha}+\sum_{i}^{N}\left[r \sum_{\alpha}^{n} \sigma_{i \alpha}^{2}+\frac{B_{i j}}{2}\left(\sum_{\alpha}^{n} \sigma_{i \alpha}^{2}\right)^{2}\right]
$$

where $r$ serves as a variable for Laplace transform (in getting the partition function for the walk) and its value needs not to be specified in our discussion. Further detail of this correspondence can be found in [55].

Comparing this Hamiltonian with the one considered in spin glass dynamics [8], in terms of soft-spin,

$$
H=\frac{1}{2} \sum_{\langle i j\rangle}\left(r_{0} \delta_{i j}-2 \beta J_{i j}\right) \sigma_{i} \sigma_{j}+u \sum_{i} \sigma_{i}^{4}-\sum_{i} h_{i} \sigma_{i}
$$

we see that the main difference is where the randomness enters. Eq. (5.2) has the quenched randomness $J_{i j}$ being a two-spin coupling, while the random coupling $B_{i j}$ in Eq. (5.1) is in 4-spin term. Note that the Hamiltonian (5.1) is also different from the $p$-spin-interaction spin glass studied in Ref. [9],

$$
H=-\sum_{i_{1}<i_{2} \cdots<i_{p}} J_{i_{1} \cdots i_{p}} \sigma_{i_{1}} \cdots \sigma_{i_{p}}-\sum_{i=1}^{N} h_{i} \sigma_{i}
$$

(or, in terms of soft-spin,

$$
H=\sum_{i}\left[\frac{r_{0}}{2} \alpha_{i}^{2}+u \sigma_{i}^{4}\right]-\beta \sum J_{i_{1} \cdots i_{p}} \sigma_{i_{1}} \cdots \sigma_{i_{p}}-\beta \sum_{i=1}^{N} h_{i} \sigma_{i}
$$

). Although Eq. (5.4) appears to be very similar to Eq. (5.1), the additional two-spin coupling $J_{i j}$ and the $O(n)$-symmetry preserving term $B_{i j}$ in Eq. (5.1) make the story entirely different. It is unclear, and seems unlikely, that the (qualitative) dynamic features obtained in Refs. [8.9] have their counterparts in the dynamics of Eq. (5.1). In fact, we are not aware of any study of Eq. (5.1) itself as a possible spin glass model. Perhaps Eq. (5.1) being a spin glass model has not been studied, either statically or dynamically, before. 
(Perhaps the ${ }^{3} \mathrm{He}-{ }^{4} \mathrm{He}$-aerogel system mentioned in Introduction is more relevant to the heteropolymer solution than spin glass system. We argued in the previous section that the randomness in heteropolymer solution is not completely quenched in space. In ${ }^{3} \mathrm{He}-{ }^{4} \mathrm{He}-$ aerogel system, according to the picture proposed by Chan and coworkers [2], at low ${ }^{4} \mathrm{He}$ concentration, "He atoms can be seen as "partially quenched": Some are bound onto the aerogel, being completely quenched, while some are in freely moving superfluid phase. This interplay of quenched randomness and annealed randomness has been theoretically studied by Maritan et. al. [56], using the so-called Blume-Emery-Griffiths (BEG) Hamiltonian [57]. Interestingly, the similarity between the BEG Hamiltonian [56,57] and Eq. (5.1) seems higher than Eqs. (5.2) and (5.4).)

The above observation is only at the level of the Hamiltonian used in each model. In fact, more significant difference at the level of kinetic equation exists. In the Oono-Freed kinetic equation (2.4) the dynamical variables are monomer positions $\left\{\mathbf{r}_{i}\right\}$ and solvent velocity field u. On the other hand, the dynamical variables used in Refs. 88,9] are spin components $\left\{\sigma_{i}\right\}$. When $n \rightarrow 0$, spins $\left\{\sigma_{i}\right\}$ do not become monomer positions $\left\{\mathbf{r}_{i}\right\}$ and solvent velocity

field u. Although the effective equation of motion does share some common features, e.g., memory effect, renormalized noise, etc., with the ones found in spin glass study, these are the features common to the machinery, functional integral formalism plus mean field approximation. Having these common features still allows entirely different physics. As we argued at the end of Sec. IVA, our system is somewhat intermediate between the solid and molten phases of dilute magnets, and is perhaps more similar to ${ }^{3} \mathrm{He}^{4}{ }^{4} \mathrm{He}$ mixture in porous media than spin glasses.

\section{CONCLUSION}

The main result of this paper is the effective equation of motion Eqs. (3.13) and (3.27) and the renormalized relaxation spectrum.

We first ask how adaptive our model is. In polymer dynamics much work has been done 
on homopolymers. There also have been some work on the dynamics of copolymers. As a first approximation, if the heterogeneity of the chain is not important, homopolymer is a reasonable model. It is seen in the above calculation that the lowest order effect of the heterogeneity parameter $B$ is proportional to $B^{2}$. This also indicates that this approximation makes sense. It is also known that the natural-occurred heteropolymers such as proteins are composed of at most 20 types of monomers (amino acids). In this sense the approximation of independent interaction energies $B_{i j}$ employed in this paper may be reasonable: It was shown [4] that the greater the number of monomer types is, the better the approximation of independent $B_{i j}$ is.

Furthermore, in a recent simulation [58, it is shown that in the early stage of the collapse of homopolymer, from coil state to globule state, some clusters form along the chain. One way to see this process is to view the chain, with clusters of various sizes, as an effective heteropolymer chain formed by monomers and clusters. This is plausible if one uses a coarsegrained viewpoint. One may argue that these clusters are not stable; they constantly form and annihilate. However, on average and in a coarse-grained sense, this constant birthand-death of clusters probably can be ignored because the clusters seldom grow larger than certain scale, as the simulation shows. We may take the upper length scale of these clusters as a basic length scale in the model, and treat the clusters as heterogeneous monomers. These clusters may have different sizes and masses, which are also allowed in our model because sizes and masses of monomers do not appear in the Oono-Freed kinetic equation. In this sense, our model can also serve as a model for a homopolymer chain in poor solvent during its early stage of collapse.

Ideally, similar to Ref. [17], the next calculation would be applying renormalization group technique to find the scaling form of the relaxation spectrum. This does not seem promising, however. To perform this calculation, we need an ansatz for the $\omega$-dependence of $\boldsymbol{G}(\omega, \mathbf{k})$, analogous to the one used in spin glass dynamics [8], as well as an ansatz for its k-dependence. Nevertheless, we do not expect that the renormalization group calculation, even if it is possible, will change the conclusion, regarding the dynamically null effect of the 
time-persistent part of response function, reached in Sec. IVB.

The most important development of the present model would be to incorporate chain compactisation, i.e. consider the dynamics of the heteropolymer globule. Another relevant and important aspect of polymer dynamics which may be studied along these lines is kinetics of coil-globule transitions [59] which may be relevant also for protein folding.

\section{ACKNOWLEDGMENTS}

One of us (J.-R.R.) would like to thank Prof. Y. Oono on discussion regarding App. A and Ref. [37].

\section{APPENDIX A: DERIVATION OF KIRKWOOD DIFFUSION EQUATION}

We show here that the Kirkwood diffusion equation can be derived directly without the projection technique used in Refs. [12,16

If the Hamiltonian is the Edwards Hamiltonian, the equation of motion derived from Eqs. (2.4) will be (cf. Eq. (2.10)

$$
\begin{gathered}
\frac{\partial}{\partial t} \mathbf{r}_{i}(t)=-\frac{1}{\zeta_{0}} \frac{\partial H_{E}}{\partial \mathbf{r}_{i}(t)}+g_{0} \mathbf{u}_{0}^{E}\left(\mathbf{r}_{i}(t), t\right)+\boldsymbol{\theta}_{0 i}(t)+g_{0} \mathbf{u}_{R}^{E}\left(\mathbf{r}_{i}(t), t\right) \\
\mathbf{u}_{0}^{E}(\mathbf{r}, t)=\int_{\mathbf{k}} e^{i \mathbf{k} \cdot \mathbf{r}} \int_{-\infty}^{t} d t^{\prime} e^{-\nu_{0} k^{2}\left(t-t^{\prime}\right)} \mathbf{P}^{T}(\hat{\mathbf{k}}) \cdot\left[-\frac{g_{0}}{\rho_{0}} \sum_{i=0}^{N-1} \frac{\partial H_{E}}{\partial \mathbf{r}_{i}\left(t^{\prime}\right)} e^{-i \mathbf{k} \cdot \mathbf{r}_{i}\left(t^{\prime}\right)}\right], \\
\mathbf{u}_{R}^{E}(\mathbf{r}, t)=\int_{\mathbf{k}} e^{i \mathbf{k} \cdot \mathbf{r}} \int_{-\infty}^{t} d t^{\prime} e^{-\nu_{0} k^{2}\left(t-t^{\prime}\right)} \mathbf{P}^{T}(\hat{\mathbf{k}}) \cdot \boldsymbol{\xi}_{0}\left(\mathbf{k}, t^{\prime}\right)
\end{gathered}
$$

When Markov approximation is applicable,

$$
e^{-\nu_{0} k^{2}\left(t-t^{\prime}\right)} \rightarrow \frac{2}{\nu_{0} k^{2}} \delta\left(t-t^{\prime}\right)
$$

the equation of motion becomes

$$
\frac{\partial}{\partial t} \mathbf{r}_{i}(t)=-\frac{1}{\zeta_{0}} \frac{\partial H_{E}}{\partial \mathbf{r}_{i}(t)}-g_{0}^{2} \sum_{j} \mathbf{T}\left(\mathbf{r}_{i}(t), \mathbf{r}_{j}(t)\right) \frac{\partial H_{E}}{\partial \mathbf{r}_{j}(t)}+\boldsymbol{\theta}_{0 i}(t)+g_{0} \int_{\mathbf{k}} e^{i \mathbf{k} \cdot \mathbf{r}_{i}(t)} \frac{1}{\eta_{0} k^{2}} \mathbf{P}^{T}(\hat{\mathbf{k}}) \cdot \boldsymbol{\xi}(\mathbf{k}, t),
$$


Eq. (A5) is a Langevin equation with noise $\boldsymbol{\theta}^{E}\left(\mathbf{r}_{i}(t), t\right) \equiv \boldsymbol{\theta}_{0 i}(t)+g_{0} \mathbf{u}_{R}^{E}\left(\mathbf{r}_{i}(t), t\right)$ satisfying

$$
\left\langle\boldsymbol{\theta}^{E}\left(\mathbf{r}_{i}(t), t\right) \boldsymbol{\theta}^{E}\left(\mathbf{r}_{j}\left(t^{\prime}\right), t^{\prime}\right)\right\rangle=2 \zeta_{0} \delta\left(t-t^{\prime}\right) \delta_{i j} \mathbf{1}+2 g_{0}^{2} \mathbf{T}\left(\mathbf{r}_{i}(t), \mathbf{r}_{j}\left(t^{\prime}\right)\right) \delta\left(t-t^{\prime}\right)
$$

The Fokker-Planck equation corresponding to this Langevin equation is

$$
\frac{\partial}{\partial t} P\left(\left\{\mathbf{r}_{i}\right\}, t\right)=\sum_{i} \sum_{j} \frac{\partial}{\partial \mathbf{r}_{i}} \cdot\left[\zeta^{-1} \mathbf{1} \delta_{i j}+g_{0}^{2} \mathbf{T}\left(\mathbf{r}_{i}, \mathbf{r}_{j}\right)\right] \cdot\left[\frac{\partial}{\partial \mathbf{r}_{j}}+\frac{\partial H_{E}}{\partial \mathbf{r}_{j}}\right] P\left(\left\{\mathbf{r}_{i}\right\}, t\right)
$$

where $P\left(\left\{\mathbf{r}_{i}\right\}, t\right)$ is the probability distribution function of chain conformation $\left.\left\{\mathbf{r}_{i}\right\}\right)$. Eq. (A7) is exactly the Kirkwood diffusion equation.

\section{APPENDIX B: DERIVATION OF THE EFFECTIVE LAGRANGIAN}

Here we detail the calculation.

$$
\text { 1. } \sum_{i \neq j} \mathcal{O}_{i j}^{2} \text { term }
$$

Define

$$
\begin{aligned}
\bar{A}\left(t, t^{\prime}, \mathbf{R}, \mathbf{R}^{\prime}\right) & \equiv \sum_{i} a_{i}(t) a_{i}\left(t^{\prime}\right) \delta\left(\mathbf{r}_{i}(t)-\mathbf{R}\right) \delta\left(\mathbf{r}_{i}\left(t^{\prime}\right)-\mathbf{R}^{\prime}\right) \\
\bar{B}\left(t, t^{\prime}, \mathbf{R}, \mathbf{R}^{\prime}\right) & \equiv \sum_{i} \delta\left(\mathbf{r}_{i}(t)-\mathbf{R}\right) \delta\left(\mathbf{r}_{i}\left(t^{\prime}\right)-\mathbf{R}^{\prime}\right) \\
\bar{C}\left(t, t^{\prime}, \mathbf{R}, \mathbf{R}^{\prime}\right) & \equiv \sum_{i} a_{i}(t) \delta\left(\mathbf{r}_{i}(t)-\mathbf{R}\right) \delta\left(\mathbf{r}_{i}\left(t^{\prime}\right)-\mathbf{R}^{\prime}\right) \\
\bar{D}\left(t, t^{\prime}, \mathbf{R}, \mathbf{R}^{\prime}\right) & \equiv \sum_{i} a_{i}\left(t^{\prime}\right) \delta\left(\mathbf{r}_{i}(t)-\mathbf{R}\right) \delta\left(\mathbf{r}_{i}\left(t^{\prime}\right)-\mathbf{R}^{\prime}\right)
\end{aligned}
$$

Note that

$$
\begin{aligned}
& \bar{A}\left(t, t^{\prime}, \mathbf{R}, \mathbf{R}^{\prime}\right)=\bar{A}\left(t^{\prime}, t, \mathbf{R}^{\prime}, \mathbf{R}\right) \\
& \bar{B}\left(t, t^{\prime}, \mathbf{R}, \mathbf{R}^{\prime}\right)=\bar{B}\left(t^{\prime}, t, \mathbf{R}^{\prime}, \mathbf{R}\right) \\
& \bar{C}\left(t, t^{\prime}, \mathbf{R}, \mathbf{R}^{\prime}\right)=\bar{D}\left(t^{\prime}, t, \mathbf{R}^{\prime}, \mathbf{R}\right)
\end{aligned}
$$

and they are all of order $O\left(N^{2}\right)$. Then, 


$$
\begin{aligned}
\sum_{i \neq j} \mathcal{O}_{i j}^{2}=\frac{1}{2} \int d t d t^{\prime} d 1 d 2 d 3 d 4 \times & {\left[\bar{A}\left(t, t^{\prime}, 1,3\right) \bar{B}\left(t, t^{\prime}, 2,4\right)+\bar{A}\left(t, t^{\prime}, 2,3\right) \bar{B}\left(t, t^{\prime}, 1,4\right)\right.} \\
& +\bar{A}\left(t, t^{\prime}, 1,4\right) \bar{B}\left(t, t^{\prime}, 2,3\right)+\bar{A}\left(t, t^{\prime}, 2,4\right) \bar{B}\left(t, t^{\prime}, 1,3\right) \\
& +\bar{C}\left(t, t^{\prime}, 1,3\right) \bar{D}\left(t, t^{\prime}, 2,4\right)+\bar{C}\left(t, t^{\prime}, 2,3\right) \bar{D}\left(t, t^{\prime}, 1,4\right) \\
& \left.+\bar{C}\left(t, t^{\prime}, 1,4\right) \bar{D}\left(t, t^{\prime}, 2,3\right)+\bar{C}\left(t, t^{\prime}, 2,4\right) \bar{D}\left(t, t^{\prime}, 1,3\right)\right] \\
& \times U(1-2) U(3-4)+O(N)
\end{aligned}
$$

Let column matrix $\psi\left(t, t^{\prime}, 1,2,3,4\right)$ and $16 \times 16$ matrix $S_{1}$ be

$$
\begin{aligned}
& \psi_{A}\left(t, t^{\prime}, 1,2,3,4\right) \equiv\left(\begin{array}{c}
\bar{A}\left(t, t^{\prime}, 1,3\right) \\
\bar{A}\left(t, t^{\prime}, 2,3\right) \\
\bar{A}\left(t, t^{\prime}, 1,4\right) \\
\bar{A}\left(t, t^{\prime}, 2,4\right)
\end{array}\right), \quad \psi_{B}\left(t, t^{\prime}, 1,2,3,4\right) \equiv\left(\begin{array}{c}
\bar{B}\left(t, t^{\prime}, 2,4\right) \\
\bar{B}\left(t, t^{\prime}, 1,4\right) \\
\bar{B}\left(t, t^{\prime}, 2,3\right) \\
\bar{B}\left(t, t^{\prime}, 1,3\right)
\end{array}\right), \\
& \psi_{C}\left(t, t^{\prime}, 1,2,3,4\right) \equiv\left(\begin{array}{c}
\bar{C}\left(t, t^{\prime}, 1,3\right) \\
\bar{C}\left(t, t^{\prime}, 2,3\right) \\
\bar{C}\left(t, t^{\prime}, 1,4\right) \\
\bar{C}\left(t, t^{\prime}, 2,4\right)
\end{array}\right), \quad \psi_{D}\left(t, t^{\prime}, 1,2,3,4\right) \equiv\left(\begin{array}{c}
\bar{D}\left(t, t^{\prime}, 2,4\right) \\
\bar{D}\left(t, t^{\prime}, 1,4\right) \\
\bar{D}\left(t, t^{\prime}, 2,3\right) \\
\bar{D}\left(t, t^{\prime}, 1,3\right)
\end{array}\right) \\
& \psi\left(t, t^{\prime}, 1,2,3,4\right) \equiv\left(\begin{array}{c}
\psi_{A}\left(t, t^{\prime}, 1,2,3,4\right) \\
\psi_{B}\left(t, t^{\prime}, 1,2,3,4\right) \\
\psi_{C}\left(t, t^{\prime}, 1,2,3,4\right) \\
\psi_{D}\left(t, t^{\prime}, 1,2,3,4\right)
\end{array}\right), \quad S_{1} \equiv\left(\begin{array}{cccc}
0 & 1_{4} & 0 & 0 \\
1_{4} & 0 & 0 & 0 \\
0 & 0 & 0 & 1_{4} \\
0 & 0 & 1_{4} & 0
\end{array}\right)
\end{aligned}
$$

(where $1_{4}$ is a $4 \times 4$ unit matrix), then

$$
\sum_{i \neq j} \mathcal{O}_{i j}^{2}=\frac{1}{4} \int d t d t^{\prime} d 1 d 2 d 3 d 4 \psi^{T}\left(t, t^{\prime}, 1,2,3,4\right) S_{1} \psi\left(t, t^{\prime}, 1,2,3,4\right) U(1-2) U(3-4)+O(N)
$$

\section{2. $\sum_{i \neq j} \mathcal{O}_{i j}$ term}

The calculation for $\sum_{i \neq j} \mathcal{O}_{i j}$ can be performed in a similar way. However, because of the symmetry of $\bar{C}$ and $\bar{D}$, there is an ambiguity in expressing $\sum_{i \neq j} \mathcal{O}_{i j}$ as a sum of products of 
$\bar{C}$ and $\bar{B}$, or of $\bar{C}$ and $\bar{B}$, or a mixed type. This should not make the final result different, as we expect the same symmetry can be used to convert $\bar{C}$ and $\bar{D}$.

If we express $\sum_{i \neq j} \mathcal{O}_{i j}$ in terms of products of $\bar{C}$ and $\bar{B}$, then

$$
\sum_{i \neq j} \mathcal{O}_{i j}=\frac{1}{4} \int d t d t^{\prime} d 1 d 2 d 3 d 4 \psi^{T}\left(t, t^{\prime}, 1,2,3,4\right) S_{2} \psi\left(t, t^{\prime}, 1,2,3,4\right) U(1-2) \bar{\delta}\left(t-t^{\prime}\right)+O(N)
$$

$$
S_{2} \equiv\left(\begin{array}{cccc}
0 & 0 & 0 & 0 \\
0 & 0 & 1_{4} & 0 \\
0 & 1_{4} & 0 & 0 \\
0 & 0 & 0 & 0
\end{array}\right)
$$

where the overbarred delta function $\bar{\delta}$ is used to keep track the fact that the integral over time variables, hence, the $\delta$-function, has to be done after the integral over space variables has been performed.

\section{Gaussian transform and mean-field approximation}

Combining the results from the previous two sections, $\left\langle e^{L_{R}}\right\rangle_{B}$ in Eq. (3.8) can be written as

$$
\exp \left\{\int d t d t^{\prime} d 1 d 2 d 3 d 4\left[\frac{B^{2}}{8 \zeta_{0}^{2}} \psi^{T} S_{1} \psi U(1-2) U(3-4) \frac{B_{0}}{4 \zeta_{0}} \psi^{T} S_{2} \psi U(1-2) \bar{\delta}\left(t-t^{\prime}\right)\right]+O(N)\right\}
$$

Introducing variables $Q_{1}(1,2,3,4) \cdots Q_{16}(1,2,3,4)$ and performing a Gaussian transform, the generating functional $Z_{\theta_{0} \xi_{0}}$ becomes

$$
Z_{\theta_{0} \xi_{0}}=\int\left\{\mathcal{D} \mathbf{r}_{i}\right\}\left\{\mathcal{D} \hat{\mathbf{r}}_{i}\right\}\left\{\mathcal{D} Q_{i}\right\} \exp \left[-\frac{1}{4} \mathcal{Q}^{T} \mathcal{A} \mathcal{Q}+\mathcal{Q}^{T} \psi+L_{0}+O(N)\right]
$$

where

$$
\left\{\mathcal{D} Q_{i}\right\} \equiv \prod_{i=1}^{16} \mathcal{D} Q_{i}
$$


$\mathcal{Q}$ is the column matrix formed by $Q_{1} \cdots Q_{16}$ and $\mathcal{A}$ is the coefficient of the quadratic term in Eq. (B10).

Using steepest decent method the mean-field approximation gives

$$
\begin{aligned}
& Q_{1}^{0}\left(t, t^{\prime}, 1,2,3,4\right)=\frac{B^{2}}{4 \zeta_{0}^{2}} U(1-2) U(3-4)\left\langle\bar{B}\left(t, t^{\prime}, 2,4\right)\right\rangle \\
& Q_{2}^{0}\left(t, t^{\prime}, 1,2,3,4\right)=\frac{B^{2}}{4 \zeta_{0}^{2}} U(1-2) U(3-4)\left\langle\bar{B}\left(t, t^{\prime}, 1,4\right)\right\rangle \\
& Q_{3}^{0}\left(t, t^{\prime}, 1,2,3,4\right)=\frac{B^{2}}{4 \zeta_{0}^{2}} U(1-2) U(3-4)\left\langle\bar{B}\left(t, t^{\prime}, 2,3\right)\right\rangle \\
& Q_{4}^{0}\left(t, t^{\prime}, 1,2,3,4\right)=\frac{B^{2}}{4 \zeta_{0}^{2}} U(1-2) U(3-4)\left\langle\bar{B}\left(t, t^{\prime}, 1,3\right)\right\rangle \\
& Q_{5}^{0}\left(t, t^{\prime}, 1,2,3,4\right)=\frac{B^{2}}{4 \zeta_{0}^{2}} U(1-2) U(3-4)\left\langle\bar{A}\left(t, t^{\prime}, 1,3\right)\right\rangle+\frac{B_{0}}{2 \zeta_{0}} U(1-2) \bar{\delta}\left(t-t^{\prime}\right)\left\langle\bar{C}\left(t, t^{\prime}, 1,3\right)\right\rangle \\
& Q_{6}^{0}\left(t, t^{\prime}, 1,2,3,4\right)=\frac{B^{2}}{4 \zeta_{0}^{2}} U(1-2) U(3-4)\left\langle\bar{A}\left(t, t^{\prime}, 2,3\right)\right\rangle+\frac{B_{0}}{2 \zeta_{0}} U(1-2) \bar{\delta}\left(t-t^{\prime}\right)\left\langle\bar{C}\left(t, t^{\prime}, 2,3\right)\right\rangle \\
& Q_{7}^{0}\left(t, t^{\prime}, 1,2,3,4\right)=\frac{B^{2}}{4 \zeta_{0}^{2}} U(1-2) U(3-4)\left\langle\bar{A}\left(t, t^{\prime}, 1,4\right)\right\rangle+\frac{B_{0}}{2 \zeta_{0}} U(1-2) \bar{\delta}\left(t-t^{\prime}\right)\left\langle\bar{C}\left(t, t^{\prime}, 1,4\right)\right\rangle \\
& Q_{8}^{0}\left(t, t^{\prime}, 1,2,3,4\right)=\frac{B^{2}}{4 \zeta_{0}^{2}} U(1-2) U(3-4)\left\langle\bar{A}\left(t, t^{\prime}, 2,4\right)\right\rangle+\frac{B_{0}}{2 \zeta_{0}} U(1-2) \bar{\delta}\left(t-t^{\prime}\right)\left\langle\bar{C}\left(t, t^{\prime}, 2,4\right)\right\rangle \\
& Q_{9}^{0}\left(t, t^{\prime}, 1,2,3,4\right)=\frac{B^{2}}{4 \zeta_{0}^{2}} U(1-2) U(3-4)\left\langle\bar{D}\left(t, t^{\prime}, 2,4\right)\right\rangle+\frac{B_{0}}{2 \zeta_{0}} U(1-2) \bar{\delta}\left(t-t^{\prime}\right)\left\langle\bar{B}\left(t, t^{\prime}, 2,4\right)\right\rangle \\
& Q_{10}^{0}\left(t, t^{\prime}, 1,2,3,4\right)=\frac{B^{2}}{4 \zeta_{0}^{2}} U(1-2) U(3-4)\left\langle\bar{D}\left(t, t^{\prime}, 1,4\right)\right\rangle+\frac{B_{0}}{2 \zeta_{0}} U(1-2) \bar{\delta}\left(t-t^{\prime}\right)\left\langle\bar{B}\left(t, t^{\prime}, 1,4\right)\right\rangle \\
& Q_{11}^{0}\left(t, t^{\prime}, 1,2,3,4\right)=\frac{B^{2}}{4 \zeta_{0}^{2}} U(1-2) U(3-4)\left\langle\bar{D}\left(t, t^{\prime}, 2,3\right)\right\rangle+\frac{B_{0}}{2 \zeta_{0}} U(1-2) \bar{\delta}\left(t-t^{\prime}\right)\left\langle\bar{B}\left(t, t^{\prime}, 2,3\right)\right\rangle \\
& Q_{12}^{0}\left(t, t^{\prime}, 1,2,3,4\right)=\frac{B^{2}}{4 \zeta_{0}^{2}} U(1-2) U(3-4)\left\langle\bar{D}\left(t, t^{\prime}, 1,3\right)\right\rangle+\frac{B_{0}}{2 \zeta_{0}} U(1-2) \bar{\delta}\left(t-t^{\prime}\right)\left\langle\bar{B}\left(t, t^{\prime}, 1,3\right)\right\rangle \\
& Q_{13}^{0}\left(t, t^{\prime}, 1,2,3,4\right)=\frac{B^{2}}{4 \zeta_{0}^{2}} U(1-2) U(3-4)\left\langle\bar{C}\left(t, t^{\prime}, 1,3\right)\right\rangle \\
& Q_{14}^{0}\left(t, t^{\prime}, 1,2,3,4\right)=\frac{B^{2}}{4 \zeta_{0}^{2}} U(1-2) U(3-4)\left\langle\bar{C}\left(t, t^{\prime}, 2,3\right)\right\rangle \\
& Q_{15}^{0}\left(t, t^{\prime}, 1,2,3,4\right)=\frac{B^{2}}{4 \zeta_{0}^{2}} U(1-2) U(3-4)\left\langle\bar{C}\left(t, t^{\prime}, 1,4\right)\right\rangle \\
& Q_{16}^{0}\left(t, t^{\prime}, 1,2,3,4\right)=\frac{B^{2}}{4 \zeta_{0}^{2}} U(1-2) U(3-4)\left\langle\bar{C}\left(t, t^{\prime}, 2,4\right)\right\rangle
\end{aligned}
$$

where the angular bracket means 


$$
\langle\cdots\rangle=\frac{\int\left\{\mathcal{D} \mathbf{r}_{i}\right\}\left\{\mathcal{D} \hat{\mathbf{r}}_{i}\right\}(\cdots) e^{L_{0}} e^{\mathcal{Q}^{T} \psi}}{\int\left\{\mathcal{D} \mathbf{r}_{i}\right\}\left\{\mathcal{D} \hat{\mathbf{r}}_{i}\right\} e^{L_{0}} e^{\mathcal{Q}^{T} \psi}}
$$

The mean-field solution for $Q_{i}^{0}$ are determined self-consistently from these equations.

Therefore, the effective Lagrangian $L_{e} \equiv L_{0}+\left(\mathcal{Q}^{0}\right)^{T} \psi$ is

$$
\begin{aligned}
L_{e}=L_{0}+\frac{B^{2}}{\zeta_{0}^{2}} \int d t d t^{\prime} d 1 d 2 d 3 d 4 U(1-2) U(3-4)\left[\left\langle\bar{A}\left(t, t^{\prime}, 1,3\right)\right\rangle \bar{B}\left(t, t^{\prime}, 2,4\right)\right. \\
\left.+\left\langle\bar{B}\left(t, t^{\prime}, 2,4\right)\right\rangle \bar{A}\left(t, t^{\prime}, 1,3\right)+2\left\langle\bar{C}\left(t, t^{\prime}, 1,3\right)\right\rangle \bar{D}\left(t, t^{\prime}, 2,4\right)\right] \\
+\frac{2 B_{0}}{\zeta_{0}} \int d t d t^{\prime} d 1 d 2 d 3 d 4 \delta\left(t-t^{\prime}\right) U(1-2)\left[\left\langle\bar{C}\left(t, t^{\prime}, 1,3\right)\right\rangle \bar{B}\left(t, t^{\prime}, 2,4\right)\right. \\
\left.+\left\langle\bar{B}\left(t, t^{\prime}, 2,4\right)\right\rangle \bar{C}\left(t, t^{\prime}, 1,3\right)\right]
\end{aligned}
$$

\section{APPENDIX C: FIRST ORDER CORRECTION OF RELAXATION SPECTRUM}

The equation of motion in Sec. IVB has the following form

$$
G_{0}^{-1}(p, \omega) \boldsymbol{\xi}(p, \omega)=\boldsymbol{\mu}(p, \omega)+\boldsymbol{F}[\boldsymbol{\xi}, p, \omega]
$$

Substitute the zeroth order solution

$$
G_{0}^{-1}(p, \omega) \boldsymbol{\xi}^{(0)}(p, \omega)=\boldsymbol{\mu}(p, \omega)
$$

into it, the first order solution is

$$
\boldsymbol{\xi}^{(1)}(p, \omega)=G_{0}(p, \omega) \boldsymbol{\mu}(p, \omega)+G_{0}(p, \omega) \boldsymbol{F}\left[\boldsymbol{\xi}^{(0)}, p, \omega\right]
$$

This solution contains terms to the lowest nontrivial order. Since $\boldsymbol{\xi}$ enters in $\boldsymbol{F}$ through exponential function, further iteration does not change the result to the lowest nontrivial order. Therefore, to the order we seek for, the solution is

$$
\boldsymbol{\xi}(p, \omega)=G_{0}(p, \omega) \boldsymbol{\mu}(p, \omega)+G_{0}(p, \omega) \boldsymbol{F}\left[\boldsymbol{\xi}^{(0)}, p, \omega\right]+\text { higher orders in }
$$

To find the relaxation spectrum, we find the average (over $\boldsymbol{\mu}$ of the following tensor product 


$$
\begin{aligned}
\boldsymbol{\xi}(p, \omega) \boldsymbol{\xi}\left(p^{\prime}, \omega^{\prime}\right) & =G_{0}(p, \omega) G_{0}\left(p^{\prime}, \omega^{\prime}\right) \boldsymbol{\mu}(p, \omega) \boldsymbol{\mu}\left(p^{\prime}, \omega^{\prime}\right) \\
& +G_{0}(p, \omega) G_{0}\left(p^{\prime}, \omega^{\prime}\right)\left[\boldsymbol{\mu}(p, \omega) \boldsymbol{F}^{(0)}\left(p^{\prime}, \omega^{\prime}\right)+\boldsymbol{\mu}\left(p^{\prime}, \omega^{\prime}\right) \boldsymbol{F}^{(0)}(p, \omega)\right]
\end{aligned}
$$

where $\boldsymbol{F}^{(0)}(p, \omega) \equiv \boldsymbol{F}\left[\boldsymbol{\xi}^{(0)}, p, \omega\right]$, and the term containing two $\boldsymbol{F}^{(0)}(p, \omega)$ has been dropped because $\boldsymbol{F}^{(0)}(p, \omega)$ is at least the lowest nontrivial order.

Let

$$
\begin{gathered}
B_{N}\left(\tau, \tau^{\prime}\right) \equiv \frac{1}{N \zeta_{0}} \sum_{p} \frac{Q_{p}^{2}}{\Lambda_{p}} \\
B_{N}^{B}\left(\tau, t, t^{\prime}\right) \equiv \frac{2}{N} \sum_{p} \frac{\cos ^{2} \frac{p \pi \tau}{N}}{\zeta_{0} \Lambda_{p}}\left(1-e^{-\Lambda_{p}\left|t-t^{\prime}\right|}\right)
\end{gathered}
$$

We find

$$
\begin{gathered}
\left\langle\boldsymbol{\xi}^{(0)}(p, \omega) \exp \left[i \sqrt{\frac{2}{N}} \mathbf{k} \cdot \sum_{p^{\prime}} \int_{\omega^{\prime}} e^{-i \omega t^{\prime}} \boldsymbol{\xi}\left(p^{\prime}, \omega^{\prime}\right) Q_{p^{\prime}}\left(\tau, \tau^{\prime}\right)\right]\right\rangle= \\
G_{0}(p, \omega) G_{0}(p,-\omega) \frac{2}{\zeta_{0}} \sqrt{\frac{2}{N}} i \mathbf{k} e^{i \omega t} Q_{p}\left(\tau, \tau^{\prime}\right) e^{-k^{2} B_{N}\left(\tau, \tau^{\prime}\right)} \\
\left\langle\boldsymbol{\xi}^{(0)}(p, \omega) \boldsymbol{\xi}\left(p^{\prime}, \omega^{\prime}\right) \exp \left[i \sqrt{\frac{2}{N}} \mathbf{k} \cdot \sum_{p^{\prime \prime}} \int_{\omega^{\prime \prime}} e^{-i \omega^{\prime \prime} t} \boldsymbol{\xi}^{(0)}\left(p^{\prime \prime}, \omega^{\prime \prime}\right) Q_{p^{\prime \prime}}\left(\tau, \tau^{\prime}\right)\right]\right\rangle= \\
G_{0}(p, \omega) G_{0}(p,-\omega) \frac{2}{\zeta_{0}}\left[2 \pi \delta\left(\omega+\omega^{\prime}\right) \delta_{p, p^{\prime}} \mathbf{1}-\right. \\
\left.G_{0}\left(p^{\prime}, \omega^{\prime}\right) G_{0}\left(p^{\prime},-\omega^{\prime}\right) \frac{2}{\zeta_{0}^{2}} \frac{2}{N} \mathbf{k} \mathbf{k} e^{i\left(\omega+\omega^{\prime}\right) t} Q_{p}\left(\tau, \tau^{\prime}\right) Q_{p^{\prime}}\left(\tau, \tau^{\prime}\right)\right] e^{-k^{2} B_{N}\left(\tau, \tau^{\prime}\right)} \\
\left\langle\boldsymbol{\xi}^{(0)}(p, \omega) \exp \left[i \sqrt{\frac{2}{N}} \mathbf{k} \cdot \sum_{p^{\prime}} \int_{\omega^{\prime}} \boldsymbol{\xi}\left(p^{\prime}, \omega^{\prime}\right) \cos \frac{p^{\prime} \pi \tau}{N}\left(e^{-i \omega^{\prime} t}-e^{-i \omega^{\prime} t^{\prime}}\right)\right\rangle=\right. \\
G_{0}(p, \omega) G_{0}(p,-\omega) \frac{2}{\zeta_{0}} \sqrt{\frac{2}{N}} i \mathbf{k}\left(e^{i \omega t}-e^{i \omega t^{\prime}}\right) \cos \frac{p \pi \tau}{N} e^{-k^{2} B_{N}^{B}\left(\tau, t, t^{\prime}\right)}
\end{gathered}
$$

As noted in Sec. IVA we confine ourselves to the stationary case. Therefore,

$$
\begin{aligned}
& \boldsymbol{G}\left(t, t^{\prime}, \mathbf{k}\right)=\boldsymbol{G}\left(t-t^{\prime}, \mathbf{k}\right) \\
& C\left(t, t^{\prime}, \mathbf{k}\right)=C\left(t-t^{\prime}, \mathbf{k}\right)
\end{aligned}
$$


where, assuming $\boldsymbol{G}\left(t, t^{\prime}, 1,2\right)=\boldsymbol{G}\left(t, t^{\prime}, 1-2\right)$ and $C\left(t, t^{\prime}, 1,2\right)=C\left(t, t^{\prime}, 1-2\right)$, i.e. translational invariance,

$$
\begin{aligned}
& \boldsymbol{G}\left(t, t^{\prime}, \mathbf{k}\right) \equiv \int d \mathbf{R} \boldsymbol{G}\left(t, t^{\prime}, \mathbf{R}\right) e^{-i \mathbf{k} \cdot \mathbf{R}} \\
& C\left(t, t^{\prime}, \mathbf{k}\right) \equiv \int d \mathbf{R} C\left(t, t^{\prime}, \mathbf{R}\right) e^{-i \mathbf{k} \cdot \mathbf{R}}
\end{aligned}
$$

Defining

$$
\begin{aligned}
\tilde{\boldsymbol{G}}(\tau, \omega, \mathbf{k}) & \equiv \int d t e^{i \omega t} \mathbf{G}(t, \mathbf{k}) e^{-k^{2} B_{N}^{B}(\tau, t)} \\
\left(B_{N}^{B}\left(\tau, t, t^{\prime}\right)=B_{N}^{B}\left(\tau, t-t^{\prime}\right)\right) & \text { and } \\
\tilde{C}(\tau, \omega, \mathbf{k}) & \equiv \int d t e^{i \omega t} C(t, \mathbf{k}) e^{-k^{2} B_{N}^{B}(\tau, t)}
\end{aligned}
$$

then ( $d$ is the dimensionality)

$$
\begin{gathered}
\frac{\left\langle\boldsymbol{\xi}(p, \omega) \boldsymbol{\xi}\left(p^{\prime}, \omega^{\prime}\right)\right\rangle}{2 \pi \delta\left(\omega+\omega^{\prime}\right) G_{0}(p, \omega) G_{0}(p,-\omega)} \\
=\frac{2}{\zeta_{0}} \delta_{p, p^{\prime}} \mathbf{1}+2 g_{0}^{2} \frac{2}{N} \int d \tau d \tau^{\prime} \int_{\mathbf{k}} \frac{1-1 / d}{\eta_{0} k^{2}} \cos \frac{p \pi \tau}{N} \cos \frac{p^{\prime} \pi \tau^{\prime}}{N} e^{-k^{2} B_{N}\left(\tau, \tau^{\prime}\right)} \mathbf{1} \\
+\frac{B^{2}}{2 \zeta_{0}} \frac{2}{N} \int d \tau \int_{\mathbf{k}} \mathbf{k} \mathbf{k} \cos \frac{p \pi \tau}{N} \cos \frac{p^{\prime} \pi \tau^{\prime}}{N} \tilde{C}(\tau, \omega, \mathbf{k}) \\
+\frac{2}{N} \frac{2}{\zeta_{0}} G_{0}(p, \omega) \mathcal{R}\left(p, p^{\prime}, \omega\right)+\frac{2}{N} \frac{2}{\zeta_{0}} G_{0}\left(p^{\prime},-\omega\right) \mathcal{R}\left(p^{\prime}, p,-\omega\right) \\
\mathcal{R}\left(p, p^{\prime}, \omega\right) \equiv \frac{B_{0}}{\zeta_{0}} \int d \tau d \tau^{\prime} \int_{\mathbf{k}} \frac{k^{2}}{d} \cos \frac{p \pi \tau}{N} Q_{p^{\prime}}\left(\tau, \tau^{\prime}\right) e^{-k^{2} B_{N}\left(\tau, \tau^{\prime}\right)} \mathbf{1} \\
\quad-g_{0}^{2}\left(\frac{p^{\prime} \pi}{N}\right)^{2} \int d \tau d \tau^{\prime} \int_{\mathbf{k}} \frac{1-1 / d}{\eta_{0} k^{2}} \cos \frac{p \pi \tau}{N} \cos \frac{p^{\prime} \pi \tau^{\prime}}{N} e^{-k^{2} B_{N}\left(\tau, \tau^{\prime}\right)} \mathbf{1} \\
\quad \frac{B^{2}}{2 \zeta_{0}^{2}} \int d \tau \int_{\mathbf{k}} i[\tilde{\boldsymbol{G}}(\tau, 0, \mathbf{k})-\tilde{\boldsymbol{G}}(\tau, \omega, \mathbf{k})] \cdot \mathbf{k k k} \cos \frac{p^{\prime} \pi \tau}{N} \cos \frac{p \pi \tau}{N}
\end{gathered}
$$

Note that this equation is obtained after higher order terms are dropped. Therefore, it does not mean that $\boldsymbol{\xi}(p, \omega)$ is a Gaussian process.

Define

$$
\delta \Lambda_{p}^{(1)}=-\frac{B_{0}}{\zeta_{0}} \frac{2}{N} \int d \tau d \tau^{\prime} \int_{\mathbf{k}} \frac{k^{2}}{d} \cos \frac{p \pi \tau}{N} Q_{p}\left(\tau, \tau^{\prime}\right) e^{-k^{2} B_{N}\left(\tau, \tau^{\prime}\right)}
$$




$$
\begin{gathered}
\delta \Lambda_{p}^{(2)}=-\frac{g_{0}^{2}}{\eta_{0}} \frac{2}{N}\left(\frac{p \pi}{N}\right)^{2} \int d \tau d \tau^{\prime} \int_{\mathbf{k}} \frac{1-1 / d}{k^{2}} \cos \frac{p \pi \tau}{N} \cos \frac{p \pi \tau^{\prime}}{N} e^{-k^{2} B_{N}\left(\tau, \tau^{\prime}\right)} \\
\delta \Lambda_{p}^{(3)}(\omega)=-\frac{B^{2}}{2 \zeta_{0}^{2}} \frac{2}{N} \int d \tau \int_{\mathbf{k}} \cos \frac{p \pi \tau}{N} \cos \frac{p \pi \tau^{\prime}}{N} i[\tilde{\boldsymbol{G}}(\tau, 0, \mathbf{k})-\tilde{\boldsymbol{G}}(\tau, \omega, \mathbf{k})] \cdot \mathbf{k} \frac{k^{2}}{d}
\end{gathered}
$$

The scalar contraction of the tensor product gives (We take $p=p^{\prime}$ mode only because of orthogonality of modes.),

$$
\begin{aligned}
\left\langle\boldsymbol{\xi}(p, \omega) \cdot \boldsymbol{\xi}\left(p, \omega^{\prime}\right)\right\rangle= & G_{0}(p, \omega) G_{0}(p,-\omega)\left\langle\boldsymbol{\mu}(p, \omega) \cdot \boldsymbol{\mu}\left(p, \omega^{\prime}\right)\right\rangle_{0} \\
& \times\left[1-G_{0}(p, \omega)\left(\delta \Lambda_{p}^{(1)}+\delta \Lambda_{p}^{(2)}+\delta \Lambda_{p}^{(3)}(\omega)\right)\right. \\
& \left.\quad-G_{0}(p,-\omega)\left(\delta \Lambda_{p}^{(1)}+\delta \Lambda_{p}^{(2)}+\delta \Lambda_{p}^{(3)}(-\omega)\right)\right]
\end{aligned}
$$

where $\left\langle\boldsymbol{\mu}(p, \omega) \cdot \boldsymbol{\mu}\left(p, \omega^{\prime}\right)\right\rangle_{0}$ is the value obtained from Eq. (4.13) by using zeroth order solution Eq. (C2).

Therefore,

$$
\begin{gathered}
{\left[G_{0}^{-1}(p, \omega)+\delta \Lambda_{p}(\omega)\right]\left[G_{0}^{-1}(p,-\omega)+\delta \Lambda_{p}(-\omega)\right]\left\langle\boldsymbol{\xi}(p, \omega) \cdot \boldsymbol{\xi}\left(p, \omega^{\prime}\right)\right\rangle=\left\langle\boldsymbol{\mu}(p, \omega) \cdot \boldsymbol{\mu}\left(p, \omega^{\prime}\right)\right\rangle_{0}} \\
\delta \Lambda_{p}(\omega) \equiv \delta \Lambda_{p}^{(1)}+\delta \Lambda_{p}^{(2)}+\delta \Lambda_{p}^{(3)}(\omega)
\end{gathered}
$$

The relaxation spectrum is therefore renormalized

$$
\Lambda_{p} \rightarrow \Lambda_{p}+\delta \Lambda_{p}(\omega)
$$

We note that the first two corrections, $\delta \Lambda_{p}^{(1)}$ and $\delta \Lambda_{p}^{(1)}$ due to excluded volume effect and hydrodynamic effect, respectively, are the same as those found in Ref. [17. 


\section{REFERENCES}

[1] See, for example, D. R. Tilley and J. Tilley, Superfluidity and Superconductivity, 3rd ed. (Adam Hilger, New York, 1990); D. S. Fisher, M. P. A. Fisher and D. A. Huse, Phys. Rev. B43, 130 (1991).

[2] S. B. Kim, J. Ma, and M. H. W. Chan, Phys. Rev. Lett. 71, 2268 (1993); J. Ma, S. B. Kim, L. W. Hrubesh, and M. H. W. Chan, J. Low Temp. Phys. 93, 945 (1993); N. Mulders, J. Ma, S. B. Kim, J. S. Yoon, and M. H. W. Chan, J. Low Temp. Phys. 101, $95(1995)$.

[3] See, for example, K. H. Fischer and J. A. Hertz, Spin Glasses (Cambridge University Press, New York, 1991); K. Binder and A. P. Young, Rev. Mod. Phys. 58, 801 (1986).

[4] D. L. Stein, Proc. Natl. Acad. Sci. USA 82, 3670 (1985); T. Garel and H. Orland, Europhys. Lett. 6, 307 (1988); E. I. Shakhnovich and A. M. Gutin, J. Phys. A 22, 1647 (1989); E. I. Shakhnovich and A. M. Gutin, Biophys. Chem. 34, 187 (1989).

[5] S. Edwards and P. W. Anderson, J. Phys. F 5, 965 (1975).

[6] C. De Dominicis, Phys. Rev. B 18, 4913 (1978).

[7] P. C. Martin, E. D. Siggia and H. A. Rose, Phys. Rev. A 8, 423 (1973). R. V. Jensen, J. Stat. Phys. 25, 183 (1981) and references therein.

[8] H. Sompolinsky and A. Zippelius, Phys. Rev. B 25, 6860 (1982).

[9] T. R. Kirkpatrick and D. Thirumalai, Phys. Rev. B 36, 5388 (1987).

[10] M. Doi and S. F. Edwards, The Theory of Polymer Dynamics (Oxford University Press, Oxford, 1986).

[11] R. B. Bird, C. F. Curtiss, R. C. Armstrong and O. Hassager, Dynamics of Polymeric Liquids, Vol. 2 (Wiley-Interscience, New York, 1987).

[12] Y. Oono, Adv. Chem. Phys. 61, 301 (1985). 
[13] A. Jagannathan, B. Schaub and Y. Oono, Phys. Lett. A113, 341 (1985).

[14] B. Schaub, B. A. Friedman and Y. Oono, Phys. Lett. A110, 136 (1985).

[15] A. Jagannathan, Y. Oono, and B. Schaub, J. Chem. Phys. 86, 2276 (1987); H. Johannesson and B. Schaub, Phys. Rev. A35, 3571 (1987).

[16] A. Lee, P. R. Baldwin and Y. Oono, Phys. Rev. A 30, 968 (1984).

[17] D. Jasnow and M. A. Moore, J. Physique Lett. 38, L-467 (1977); G. F. Al-Noaimi, G. C. Martinez-Mekler and C. A. Wilson, J. Physique Lett. 39, L-373 (1978); Y. Shiwa and K. Kawasaki, J. Phys. C 15, 5345 (1982).

[18] J. D. Bryngelson and P. Wolynes, J. Phys. Chem. 93, 6902 (1989).

[19] K. F. Freed, Renormalization Group Theory of Macromolecules (John Wiley and Sons, New York, 1987).

[20] J. des Cloizeaux and G. Jannink, Polymers in Solution (Oxford University Press, Oxford, 1989).

[21] Y. Oono and K. F. Freed, J. Chem. Phys. 75, 1009 (1981).

[22] L. D. Landau and E. M. Lifshitz, Fluid Mechanics (Pergamon, New York, 1959).

[23] Y. Oono and P. Baldwin, Phys. Rev. A 33, 3391 (1986).

[24] C. W. J. Beenakker and P. Mazur, Phys. Fluids, 28, 767 (1985) discusses a paradox (Smoluchowski paradox) caused by this non-applicability.

[25] Y. Oono, in Polymer-Flow Interaction, AIP Conference Proceeding No. 137, edited by Y. Rabin, (American Institute of Physics, New York, 1985).

[26] K. Kawasaki and J. D. Gunton, in Progress in Liquid Physics, edited by C. A. Croxton (John Wiley and Sons, 1978).

[27] T. A. Vilgis, J. Phys. (France) I 1, 1389 (1991). 
[28] B. Schaub, D. B. Creamer and H. Johannesson, J. Phys. A 21, 1431 (1988).

[29] H. Johannesson, D. B. Creamer and B. Schaub, J. Phys. A 20, 5071 (1987).

[30] E. Frey and D. R. Nelson, J. Phys. (Paris) I, 1, 1715 (1991).

[31] P. Hänggi and P. Jung, Adv. Chem. Phys. 89, 239 (1995).

[32] J. Zinn-Justin, Quantum Field Theory and Critical Phenomena, (Oxford University Press, Oxford, 1989).

[33] R. Bausch, H. K. Jassen and H. Wagner, Z. Phys. B, 24, 113 (1976).

[34] J. Kurchan, J. Phys. (Paris) I, 2, 1333 (1992) attempts to formulate spin glass dynamics in terms of supersymmetry.

[35] S. P. Das and G. F. Mazenko, Phys. Rev. A, 34, 2265 (1986).

[36] W. D. McComb, The Physics of Fluid Turbulence, (Oxford University Press, Oxford, 1990).

[37] Y. Oono (private communication)

[38] However, G. H. Fredrickson and E. Helfand, J. Chem. Phys. 93, 2048 (1990) find that this method is useful for the study of collective dynamics of polymer solution.

[39] F. Langouche, D. Roekaerts and E. Tirapegui, Physica, A 95, 252 (1979).

[40] R. Graham, in Springer Tracts in Modern Physics, Vol. 66, Springer-Verlag, (1973).

[41] C. De Dominicis and L. Peliti, Phys. Rev. B 18, 353 (1978).

[42] B. J. West, A. R. Bulsara, K. Lindenberg, V. Seshadri, and K. E. Shuler, Physica, 97A, $211(1979)$.

[43] H. Sompolinsky, Phys. Rev. Lett. 47, 935 (1981).

[44] R. Zwanzig, J. Stat. Phys. 9, 215 (1973). 
[45] K. Kawasaki, J. Phys. 6, 1289 (1973).

[46] J. P. Boon and S. Yip, Molecular Hydrodynamics (Dover, New York, 1991); J. P. Hansen and I. R. McDonald, Theory of Simple Liquids 2nd ed. (Academic Press, London, 1986); D. Forster, Hydrodynamic Fluctuations, Broken Symmetry, and Correlation Functions, (Addison-Wesley, Reading, Massachusetts, 1990).

[47] M. C. Wang and G. E. Uhlenbeck, Rev. Mod. Phys. Phys. 17, 323 (1945).

[48] R. Zwanzig, Phys. Rev. 124, 983 (1961).

[49] L. F. Cugliandolo and J. Kurchan, J. Phys. A 27, 5749 (1994). L. F. Cugliandolo and J. Kurchan, Phil. Mag. B 71, 501 (1995). L. F. Cugliandolo, J. Kurchan and F. Ritort, Phys. Rev. B 49, 6331 (1994). A, Crisanti, H. Horner and H.-J. Sommers, Z. Phys. B 92, 257 (1993). S. Franz and M. Mézard, Europhys. Lett. 26, 209 (1994). S. Franz and M. Mézard, Physica A 210, 48 (1994). A. Baldassarri, L. F. Cugliandolo, J. Kurchan and G. Parisi, J. Phys. A 28, 1831 (1995).

[50] H. Mori and H. Fujisaka, Prog. Theore. Phys. 49, 764 (1973). K. S. J. Nordholm and R. Zwanzig, J. Stat. Phys. 11, 143 (1974).

[51] S.-K. Ma and G. F. Mazenko, Phys. Rev. B 11, 4077 (1975).

[52] S. Stepanow, J. Phys. A 17, 3041 (1984).

[53] A. M. Gutin, A. Sali, V. I. Abkevich, M. Karplus, and E. I. Shakhnovich, to be published.

[54] P. G. de Gennes, Phys. Lett. A 38, 339 (1972).

[55] V. J. Emery, Phys. Rev. B 11, 239 (1975); D. Jasnow and M. E. Fisher, Phys. Rev., B 13, 1112 (1976).

[56] A. Maritan, M. Cieplak, M. R. Swift, F. Toigo, and J. R. Banavar, Phys. Rev. Lett. 69, 221 (1992). See also, A. Falicov and A. N. Berker, Phys. Rev. Lett. 74, 426 (1995). 
[57] M. Blume, V. J. Emery, and R. B. Griffiths, Phys. Rev. A 4, 1071 (1971).

[58] A. Byrne, P. Kiernan, D. Green and K. A. Dawson, J. Chem. Phys. 102, 573 (1995).

[59] F. Ganazzoli, R. La Ferla, and G. Allegra, Macromolecules, 28, 5285 (1995). 